\title{
Hermite-Padé approximation, isomonodromic deformation and hypergeometric integral
}

\author{
Toshiyuki Mano and Teruhisa Tsuda \\ February 24, 2015 \\ Revised: March 29, 2016 (final version)
}

\begin{abstract}
We develop an underlying relationship between the theory of rational approximations and that of isomonodromic deformations. We show that a certain duality in Hermite's two approximation problems for functions leads to the Schlesinger transformations, i.e. transformations of a linear differential equation shifting its characteristic exponents by integers while keeping its monodromy invariant. Since approximants and remainders are described by block-Toeplitz determinants, one can clearly understand the determinantal structure in isomonodromic deformations. We demonstrate our method in a certain family of Hamiltonian systems of isomonodromy type including the sixth Painlevé equation and Garnier systems; particularly, we present their solutions written in terms of iterated hypergeometric integrals. An algorithm for constructing the Schlesinger transformations is also discussed through vector continued fractions.
\end{abstract}

\section{Contents}

1 From Hermite's two approximation problems to Schlesinger transformations 4

1.1 Hermite-Padé approximation (of type I) . . . . . . . . . . . . . 4

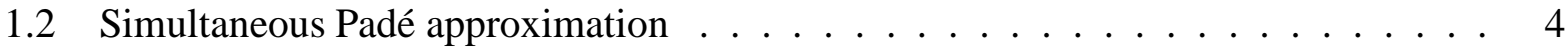

1.3 Mahler's duality in the two approximation problems . . . . . . . . . . . . 5

1.4 Schlesinger transformations . . . . . . . . . . . . . . 7

2010 Mathematics Subject Classification 34M56 (primary), 33C70, 41A21 (secondary).

Keywords: Hermite-Padé approximation, hypergeometric integral, isomonodromic deformation, Painlevé equation, vector continued fraction.

Toshiyuki Mano tmano@math.u-ryukyu.ac.jp

Department of Mathematical Science, University of the Ryukyus, Okinawa 903-0213, Japan

Teruhisa Tsuda tudateru@econ.hit-u.ac.jp

Department of Economics, Hitotsubashi University, Tokyo 186-8601, Japan 
2.1 Hermite-Padé polynomials . . . . . . . . . . . . . . . . . 10

2.2 Simultaneous Padé polynomials . . . . . . . . . . . . . . . 12

3 From vector continued fractions to Schlesinger transformations 15

3.1 Algorithm for vector continued fraction expansion . . . . . . . . . . . . . 15

3.2 Schlesinger transformations, revisited . . . . . . . . . . . . 17

4 Application to isomonodromic deformations 19

4.1 Schlesinger systems and their symmetries . . . . . . . . . . . . . 19

4.2 Polynomial Hamiltonian system $\mathcal{H}_{L, N}$ of isomonodromy type . . . . . . . . . . . 21

4.3 Solution of $\mathcal{H}_{L, N}$ in terms of hypergeometric function $F_{L, N} \ldots \ldots \ldots \ldots$

5 Solutions of $\mathcal{H}_{L, N}$ in terms of iterated hypergeometric integrals 25

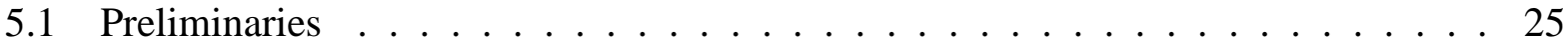

5.2 Calculation of the Schlesinger transform (I) . . . . . . . . . . . . . . 28

5.3 Calculation of the Schlesinger transform (II) . . . . . . . . . . . . . . . . 29

5.4 Calculation of the Schlesinger transform (III) . . . . . . . . . . . . . 30

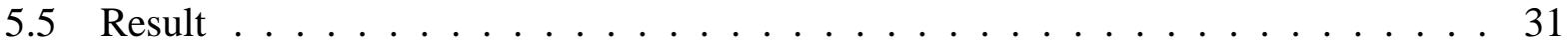

A Verification of (4.4) 33

\section{Introduction}

Let $L$ be an integer greater than one. For a given $L$-tuple of analytic functions (or formal power series) $f_{0}(w), f_{1}(w), \ldots, f_{L-1}(w)$ and of nonnegative integers $\boldsymbol{m}=\left(m_{0}, m_{1}, \ldots, m_{L-1}\right)$, Hermite considered the following two rational approximation problems. The first is to find $L$ polynomials

$$
\mathfrak{q}_{i}^{m}(w) \quad(0 \leq i \leq L-1)
$$

of degree $m_{i}-1$ such that

$$
\sum_{i=0}^{L-1} f_{i} \mathrm{q}_{i}^{\boldsymbol{m}}=O\left(w^{|\boldsymbol{m}|-1}\right),
$$

where $|\boldsymbol{m}|=\sum_{i=0}^{L-1} m_{i}$; i.e., the left-hand side has a zero of order at least $|\boldsymbol{m}|-1$ at $w=0$. The second is to find $L$ polynomials

$$
\mathfrak{p}_{i}^{m}(w) \quad(0 \leq i \leq L-1)
$$

of degree $|\boldsymbol{m}|-m_{i}$ such that

$$
f_{i} \mathfrak{p}_{j}^{\boldsymbol{m}}-f_{j} \mathfrak{p}_{i}^{\boldsymbol{m}}=O\left(w^{|\boldsymbol{m}|+1}\right) .
$$

Each system of polynomials $\left\{\mathfrak{q}_{i}^{m}\right\}$ and $\left\{p_{i}^{m}\right\}$ generically turns out to be unique up to simultaneous multiplication by constants, as an elementary consequence of linear algebra. The above approximation problems come from Hermite's study on arithmetic properties of the exponential function and are called collectively the Hermite-Padé approximations; often, the former is referred to as the 'type I' and the latter as the 'type II' or as the simultaneous Padé approximation. Note that if $L=2$ 
then both of them reduce to the (usual) Padé approximations. Although these two types of approximations were seemingly unrelated, Mahler discovered that they were fundamentally connected to each other. Put $\boldsymbol{e}_{i}=(0, \ldots, 0, \stackrel{i}{1}, 0, \ldots, 0) \in \mathbb{Z}^{L}$ for $0 \leq i \leq L-1$.

Theorem 0.1 (Mahler's duality). It holds that

$$
\left[\begin{array}{cccc}
\mathfrak{q}_{0}^{\boldsymbol{m}+e_{0}} & \mathfrak{q}_{1}^{\boldsymbol{m}+\boldsymbol{e}_{0}} & \cdots & \mathfrak{q}_{L-1}^{\boldsymbol{m}+\boldsymbol{e}_{0}} \\
\mathfrak{q}_{0}^{\boldsymbol{m}+\boldsymbol{e}_{1}} & \mathfrak{q}_{1}^{\boldsymbol{m}+\boldsymbol{e}_{1}} & \cdots & \mathfrak{q}_{L-1}^{\boldsymbol{m}+\boldsymbol{e}_{1}} \\
\vdots & \vdots & \ddots & \vdots \\
\mathfrak{q}_{0}^{\boldsymbol{m}+\boldsymbol{e}_{L-1}} & \mathfrak{q}_{1}^{\boldsymbol{m}+\boldsymbol{e}_{L-1}} & \cdots & \mathfrak{q}_{L-1}^{\boldsymbol{m}+\boldsymbol{e}_{L-1}}
\end{array}\right]\left[\begin{array}{cccc}
\mathfrak{p}_{0}^{\boldsymbol{m}-\boldsymbol{e}_{0}} & \mathfrak{p}_{0}^{\boldsymbol{m}-\boldsymbol{e}_{1}} & \cdots & \mathfrak{p}_{0}^{\boldsymbol{m}-\boldsymbol{e}_{L-1}} \\
\mathfrak{p}_{1}^{\boldsymbol{m}-\boldsymbol{e}_{0}} & \mathfrak{p}_{1}^{\boldsymbol{m}-\boldsymbol{e}_{1}} & \cdots & \mathfrak{p}_{1}^{\boldsymbol{m}-\boldsymbol{e}_{L-1}} \\
\vdots & \vdots & \ddots & \vdots \\
\mathfrak{p}_{L-1}^{\boldsymbol{m}-\boldsymbol{e}_{0}} & \mathfrak{p}_{L-1}^{\boldsymbol{m}-\boldsymbol{e}_{1}} & \cdots & \mathfrak{p}_{L-1}^{\boldsymbol{m}-\boldsymbol{e}_{L-1}}
\end{array}\right]=w^{|\boldsymbol{m}|} \cdot D
$$

with $D$ being a diagonal constant matrix. Moreover, if every diagonal part $\mathfrak{q}_{i}^{m+e_{i}}$ and $\mathfrak{p}_{i}^{m-e_{i}}$ is chosen to be a monic polynomial then $D$ becomes the identity matrix.

The aim of this paper is to develop an underlying relationship of Hermite's two approximations with the theory of linear differential equations in the complex domain, especially with that of isomonodromic deformations. Interestingly enough, Mahler's duality plays a crucial role in constructing a certain class of Schlesinger transformations, i.e. transformations of a linear differential equation shifting its characteristic exponents by integers while keeping its monodromy invariant.

In Sect. 1, we begin by introducing the two types of rational approximations for an $L$-tuple of functions, which are slightly modified (in order to fit the construction of Schlesinger transformations) from the original Hermite-Padé and simultaneous Padé approximations. We then prove a variation of Mahler's duality between them (see Theorem 1.3). Applying the approximations for the solution of an $L \times L$ Fuchsian system of linear differential equations yields its Schlesinger transformation (see Theorem 1.6); in fact, Mahler's duality guarantees the absence of apparent singularities in the new Fuchsian system after the Schlesinger transformation. In Sect. 2, we deduce determinantal representations for the approximants and remainders from the approximation conditions (see Propositions 2.1 and 2.3 and also Remark 2.2). It should be noted that any key ingredient here is written in terms of block-Toeplitz determinants. In Sect. 3, we present an algorithm for constructing the Schlesinger transformation via vector continued fraction expansions, which is a variation of the Jacobi-Perron algorithm (i.e. a higher dimensional analogue of the Euclidean algorithm).

The last two sections are devoted to the study of isomonodromic deformations. In Sect. 4 , we first review the Schlesinger system of nonlinear differential equations, which governs isomonodromic deformations of a Fuchsian system. Since a Schlesinger transformation preserves the monodromy of the Fuchsian system, it gives rise to a discrete symmetry of the corresponding Schlesinger system. We clarify, based on the above relationship with rational approximations, the determinantal structure in the general solutions of the Schlesinger systems. Next we concern a particular family of the Schlesinger systems, which possesses a unified description as a polynomial Hamiltonian system denoted by $\mathcal{H}_{L, N}(L \geq 2, N \geq 1)$; it includes various noteworthy examples of isomonodromic deformations such as the sixth Painlevé equation $\left(\mathcal{H}_{2,1}\right)$ and the Garnier system in $N$ variables $\left(\mathcal{H}_{2, N}\right)$. In Sect. 5, we demonstrate Schlesinger transformations on the previously known hypergeometric solution of $\mathcal{H}_{L, N}$ (see [25]); as a result, we obtain solutions of $\mathcal{H}_{L, N}$ written in terms of iterated hypergeometric integrals through Fubini's theorem and the Vandermonde determinant (see Theorem 5.3 and its sequel). 


\section{From Hermite's two approximation problems to Schlesinger transformations}

In this section we show how rational approximations are useful for constructing Schlesinger transformations of linear differential equations. Fix an integer $L \geq 2$. We shall first introduce two different types of rational approximation problems for an $L$-tuple

$$
f_{0}(w), f_{1}(w), \ldots, f_{L-1}(w) \in \mathbb{C} \llbracket w \rrbracket
$$

of formal power series, where we assume $f_{0}(0) \neq 0$ without loss of generality.

\subsection{Hermite-Padé approximation (of type I)}

Let $n$ be a positive integer. Consider for each $i(0 \leq i \leq L-1)$ an $L$-tuple of polynomials $Q_{j}^{(i)}=Q_{j}^{(i)}(w)(0 \leq j \leq L-1)$ of degree at most $n-1+\delta_{i, j}$, where

$$
\delta_{i, j}= \begin{cases}1 & (i=j) \\ 0 & (i \neq j)\end{cases}
$$

is the Kronecker delta. Suppose the approximation condition

$$
Q_{0}^{(i)} f_{0}+\cdots+Q_{i}^{(i)} f_{i}+w Q_{i+1}^{(i)} f_{i+1}+\cdots+w Q_{L-1}^{(i)} f_{L-1}=O\left(w^{n L}\right)
$$

is fulfilled for each $i$. This condition amounts to a system of $n L$ homogeneous linear equations for the $n L+1$ unknown coefficients of the polynomials $Q_{j}^{(i)}(0 \leq j \leq L-1)$. Under a generic condition for the power series $f_{0}, \ldots, f_{L-1}$, these polynomials are uniquely determined up to simultaneous multiplication by constants; see Sect. 2.1. We will be concerned with the row vector

$$
\widetilde{\boldsymbol{Q}}^{(i)}=\left(\widetilde{Q}_{j}^{(i)}\right)_{0 \leq j \leq L-1}=\left(Q_{0}^{(i)}, \ldots, Q_{i}^{(i)}, w Q_{i+1}^{(i)}, \ldots, w Q_{L-1}^{(i)}\right) .
$$

Remark 1.1. By construction, the polynomial $Q_{0}^{(0)}(w)$ has no constant term. Moreover, the degree of the diagonal part $Q_{i}^{(i)}(w)(0 \leq i \leq L-1)$ turns out to be $n$ exactly; see (1.4) in Sect. 1.3.

\subsection{Simultaneous Padé approximation}

We treat another type of approximation problem for the same power series $f_{0}, \ldots, f_{L-1}$. Consider for each $j(0 \leq j \leq L-1)$ an $L$-tuple of polynomials $P_{i}^{(j)}=P_{i}^{(j)}(w)(0 \leq i \leq L-1)$ of degree at most $n(L-1)-1+\delta_{i, j}$ which satisfies the following approximation conditions:

$$
\begin{array}{lll}
\text { - if } j=0 & f_{0} P_{i}^{(0)}-f_{i} P_{0}^{(0)}=O\left(w^{n L}\right) & \text { for } 1 \leq i \leq L-1 \text {; } \\
\text { • if } 1 \leq j \leq L-1 & f_{0} P_{i}^{(j)}-f_{i} P_{0}^{(j)}=O\left(w^{n L}\right) & \text { for } 1 \leq i \leq j-1, \\
& f_{0} P_{i}^{(j)}-f_{i} w P_{0}^{(j)}=O\left(w^{n L}\right) & \text { for } j \leq i \leq L-1 .
\end{array}
$$

These conditions are interpreted as a system of $n L(L-1)$ homogeneous linear equations for the $n L(L-1)+1$ unknown coefficients of the polynomials $P_{i}^{(j)}(0 \leq i \leq L-1)$. Hence the column vector

$$
\widetilde{\boldsymbol{P}}^{(j)}={ }^{\mathrm{T}}\left(\widetilde{P}_{i}^{(j)}\right)_{0 \leq i \leq L-1}={ }^{\mathrm{T}}\left(w P_{0}^{(j)}, \ldots, w P_{j-1}^{(j)}, P_{j}^{(j)}, \ldots, P_{L-1}^{(j)}\right)
$$

is generically unique up to multiplication by constants; see Sect. 2.2. 
Remark 1.2. Let $a \neq b$. It is immediate from $1 / f_{0} \in \mathbb{C} \llbracket w \rrbracket$ to verify that

- if $a, b<j \quad f_{a} \widetilde{P}_{b}^{(j)}-f_{b} \widetilde{P}_{a}^{(j)}=O\left(w^{n L+1}\right)$;

- otherwise $f_{a} \widetilde{P}_{b}^{(j)}-f_{b} \widetilde{P}_{a}^{(j)}=O\left(w^{n L}\right)$.

\subsection{Mahler's duality in the two approximation problems}

There is an interesting connection between the two approximation problems (1.1) and (1.2) although they are seemingly unrelated. The following theorem is thought of as a variation of Mahler's duality; see Theorem 0.1 or [15]. We will give a proof of it because our setup is slightly different from the original; $\mathrm{cf}$. [2, Theorem 8.1.2] and [5].

Theorem 1.3. It holds that

$$
\left[\begin{array}{c}
\widetilde{\boldsymbol{Q}}^{(0)} \\
\vdots \\
\widetilde{\boldsymbol{Q}}^{(L-1)}
\end{array}\right]\left[\widetilde{\boldsymbol{P}}^{(0)}, \ldots, \widetilde{\boldsymbol{P}}^{(L-1)}\right]=w^{n L} \cdot D
$$

with $D$ being a diagonal constant matrix.

Proof. Let us first estimate the degree of the $(i, j)$-entry

$$
M_{i j}=\sum_{k=0}^{L-1} \widetilde{Q}_{k}^{(i)} \widetilde{P}_{k}^{(j)}
$$

of the left-hand side. The degree of each polynomial $\widetilde{Q}_{k}^{(i)}$ or $\widetilde{P}_{k}^{(j)} \operatorname{reads}$

$$
\begin{aligned}
& \underbrace{Q_{0}^{(i)}, \ldots, Q_{i-1}^{(i)}}_{\operatorname{deg} \leq n-1}, \underbrace{Q_{i}^{(i)}, w Q_{i+1}^{(i)}, \ldots, w Q_{L-1}^{(i)}}_{\operatorname{deg} \leq n}, \\
& \underbrace{w P_{0}^{(j)}, \ldots, w P_{j-1}^{(j)}, P_{j}^{(j)}}_{\operatorname{deg} \leq n(L-1)}, \underbrace{P_{j+1}^{(j)}, \ldots, P_{L-1}^{(j)}}_{\operatorname{deg} \leq n(L-1)-1} .
\end{aligned}
$$

Hence we have

$$
\operatorname{deg}_{w} M_{i j} \leq \begin{cases}n L & (i \leq j) \\ n L-1 & (i>j)\end{cases}
$$

Next we shall estimate the multiplicity of $M_{i j}$ at $w=0$ by means of the approximation conditions. Consider

$$
\begin{aligned}
f_{0} M_{i j} & =f_{0} \sum_{k=0}^{L-1} \widetilde{Q}_{k}^{(i)} \widetilde{P}_{k}^{(j)} \\
& =f_{0} \widetilde{Q}_{0}^{(i)} \widetilde{P}_{0}^{(j)}+\sum_{k \neq 0} \widetilde{Q}_{k}^{(i)} f_{0} \widetilde{P}_{k}^{(j)}
\end{aligned}
$$


(i) Case $i<j$ (strictly upper triangular part)

$$
\begin{aligned}
\sum_{k \neq 0} \widetilde{Q}_{k}^{(i)} f_{0} \widetilde{P}_{k}^{(j)} & =\sum_{0<k<j} \widetilde{Q}_{k}^{(i)} f_{0} \widetilde{P}_{k}^{(j)}+\sum_{j \leq k} \widetilde{Q}_{k}^{(i)} f_{0} \widetilde{P}_{k}^{(j)} \\
& =\sum_{0<k<j} \widetilde{Q}_{k}^{(i)}\left(f_{k} \widetilde{P}_{0}^{(j)}+O\left(w^{n L+1}\right)\right)+\sum_{j \leq k} \widetilde{Q}_{k}^{(i)}\left(f_{k} \widetilde{P}_{0}^{(j)}+O\left(w^{n L}\right)\right), \quad \text { using (1.2) } \\
& =\widetilde{P}_{0}^{(j)} \sum_{k \neq 0} \widetilde{Q}_{k}^{(i)} f_{k}+O\left(w^{n L+1}\right), \quad \text { since } \widetilde{Q}_{k}^{(i)} \text { is divisible by } w \text { if } i<(j \leq) k
\end{aligned}
$$

Therefore,

$$
\begin{aligned}
f_{0} M_{i j} & =\widetilde{P}_{0}^{(j)} \sum_{k} \widetilde{Q}_{k}^{(i)} f_{k}+O\left(w^{n L+1}\right) \\
& =\widetilde{P}_{0}^{(j)} \cdot O\left(w^{n L}\right)+O\left(w^{n L+1}\right), \quad \text { using (1.1) } \\
& =O\left(w^{n L+1}\right), \quad \text { since } \widetilde{P}_{0}^{(j)} \text { is divisible by } w \text { if } 0(\leq i)<j .
\end{aligned}
$$

(ii) Case $i \geq j$ (lower triangular part)

$$
\begin{aligned}
\sum_{k \neq 0} \widetilde{Q}_{k}^{(i)} f_{0} \widetilde{P}_{k}^{(j)} & =\sum_{0<k<j} \widetilde{Q}_{k}^{(i)} f_{0} \widetilde{P}_{k}^{(j)}+\sum_{j \leq k} \widetilde{Q}_{k}^{(i)} f_{0} \widetilde{P}_{k}^{(j)} \\
& =\sum_{0<k<j} \widetilde{Q}_{k}^{(i)}\left(f_{k} \widetilde{P}_{0}^{(j)}+O\left(w^{n L+1}\right)\right)+\sum_{j \leq k} \widetilde{Q}_{k}^{(i)}\left(f_{k} \widetilde{P}_{0}^{(j)}+O\left(w^{n L}\right)\right), \quad \text { using (1.2) } \\
& =\widetilde{P}_{0}^{(j)} \sum_{k \neq 0} \widetilde{Q}_{k}^{(i)} f_{k}+O\left(w^{n L}\right) .
\end{aligned}
$$

Therefore,

$$
\begin{aligned}
f_{0} M_{i j} & =\widetilde{P}_{0}^{(j)} \sum_{k} \widetilde{Q}_{k}^{(i)} f_{k}+O\left(w^{n L}\right) \\
& =\widetilde{P}_{0}^{(j)} \cdot O\left(w^{n L}\right)+O\left(w^{n L}\right), \quad \text { using (1.1) } \\
& =O\left(w^{n L}\right) .
\end{aligned}
$$

Noticing $1 / f_{0} \in \mathbb{C} \llbracket w \rrbracket$ we verify

$$
M_{i j}= \begin{cases}O\left(w^{n L+1}\right) & (i<j) \\ O\left(w^{n L}\right) & (i \geq j) .\end{cases}
$$

Combining this with (1.3), we can conclude that $\left(M_{i j}\right)=w^{n L} \cdot D$.

Consequently, the diagonal entry $M_{i i}$ coincides with the term of highest degree in $Q_{i}^{(i)}(w) P_{i}^{(i)}(w)$ and thus

$$
\operatorname{deg}_{w} Q_{i}^{(i)}=n, \quad \operatorname{deg}_{w} P_{i}^{(i)}=n(L-1) .
$$

We henceforth normalize $\widetilde{\boldsymbol{Q}}^{(i)}$ and $\widetilde{\boldsymbol{P}}^{(j)}$ so that their diagonal parts $Q_{i}^{(i)}$ and $P_{j}^{(j)}$ become monic polynomials, i.e.

$$
\left.z^{n} Q_{i}^{(i)}\left(z^{-1}\right)\right|_{z=0}=\left.z^{n(L-1)} P_{i}^{(i)}\left(z^{-1}\right)\right|_{z=0}=1
$$

and thereby $D=I$ (the identity matrix). 
Corollary 1.4. The polynomial matrix

$$
R(z)=z^{n}\left[\begin{array}{c}
\widetilde{\boldsymbol{Q}}^{(0)}\left(z^{-1}\right) \\
\vdots \\
\widetilde{\boldsymbol{Q}}^{(L-1)}\left(z^{-1}\right)
\end{array}\right] \in \mathbb{C}^{L \times L}[z]
$$

satisfies

(i) $R(z)^{-1}=z^{n(L-1)}\left[\widetilde{\boldsymbol{P}}^{(0)}\left(z^{-1}\right), \ldots, \widetilde{\boldsymbol{P}}^{(L-1)}\left(z^{-1}\right)\right]$;

(ii) $\operatorname{det} R(z)=1$, i.e. $R(z) \in \operatorname{SL}(L, \mathbb{C}[z])$.

Proof. Theorem 1.3 shows (i) immediately. Then, it holds that $R \in \operatorname{GL}(L, \mathbb{C}[z])$ and thus $\operatorname{det} R \in$ $\mathbb{C} \backslash\{0\}$. By definition, $R$ takes the form

$$
R=\left[\begin{array}{ccc}
1 & \cdots & * \\
& \ddots & \vdots \\
& & 1
\end{array}\right]+O(z)
$$

namely, its constant term is an upper triangular matrix whose diagonal entries are all one. Therefore, we have $\operatorname{det} R=1$.

\subsection{Schlesinger transformations}

Consider an $L \times L$ Fuchsian system

$$
\frac{\mathrm{d} Y}{\mathrm{~d} z}=A Y=\sum_{i=0}^{N+1} \frac{A_{i}}{z-u_{i}} Y \quad\left(A_{i}: \text { constant matrix }\right)
$$

of linear ordinary differential equations with $N+3$ regular singularities

$$
S=\left\{u_{0}=1, u_{1}, \ldots, u_{N}, u_{N+1}=0, u_{N+2}=\infty\right\} \subset \mathbb{P}^{1}=\mathbb{C} \cup\{\infty\}
$$

on the Riemann sphere. Let $A_{N+1}$ and $A_{N+2}=-\sum_{i=0}^{N+1} A_{i}$ be upper and lower triangular matrices, respectively. Assume for simplicity there is no integer difference among the characteristic exponents $\left\{\varepsilon_{0, j}\right\}_{0 \leq j \leq L-1}$ at $z=0$ (resp. $\left\{\varepsilon_{\infty, j}\right\}_{0 \leq j \leq L-1}$ at $z=\infty$ ), i.e. the eigenvalues of the residue matrix $A_{N+1}\left(\right.$ resp. $\left.A_{N+2}\right)$. Then we have a solution $Y=Y(z)$ of (1.7) normalized as

$$
\begin{aligned}
Y & =\left(\left[\begin{array}{rrr}
1 & \cdots & * \\
& \ddots & \vdots \\
& & 1
\end{array}\right]+O(z)\right) \cdot \operatorname{diag}\left(z^{\varepsilon_{0, j}}\right)_{0 \leq j \leq L-1} \\
& =\Phi(w) \cdot \operatorname{diag}\left(w^{\varepsilon_{\infty, j}}\right)_{0 \leq j \leq L-1} \cdot C
\end{aligned}
$$

with $w=1 / z$ and $C$ being an invertible constant matrix (the connection matrix between $z=0$ and $z=\infty)$. Here $\Phi(w)$ is a matrix function holomorphic at $w=0(z=\infty)$ and $\Phi(0)$ is invertible and lower triangular, i.e.

$$
\Phi(w)=\left[\begin{array}{ccc}
* & & \\
\vdots & \ddots & \\
* & \cdots & *
\end{array}\right]+O(w)
$$


Remark 1.5. Many literatures adopt a different normalization such that the residue matrix at $z=\infty$ becomes diagonal. Our present normalization treats the two points $z=0$ and $z=\infty$ equally and it emerges naturally from the similarity reduction of the UC hierarchy, which is a context of infinitedimensional integrable systems; see [23, 24, 26]. Furthermore, as clarified by Haraoka [6], this normalization is effective to find a 'good' coordinate of the space of Fuchsian systems having a given Riemann scheme.

An analytic continuation along a loop on $\mathbb{P}^{1} \backslash S$ based at some point $z_{0}$ induces a linear transformation of $Y$ according to its multi-valuedness at the branch points $S$. We thus obtain an $L$ dimensional representation of the fundamental group $\pi_{1}\left(\mathbb{P}^{1} \backslash S ; z_{0}\right)$, which is called the monodromy of $Y$. A left multiplication $Y \mapsto \hat{Y}=R Y$ of a rational function matrix $R=R(z)$ is said to be a Schlesinger transformation if the new equation

$$
\frac{\mathrm{d} \hat{Y}}{\mathrm{~d} z}=\hat{A} \hat{Y}
$$

satisfied by $\hat{Y}$ becomes the same form as the original (1.7). Because $R(z)$ is rational, $\hat{Y}$ and $Y$ have the same monodromy though they have different characteristic exponents by integers. It is known that if we specify an admissible discrete change of the characteristic exponents then the corresponding rational function matrix $R$ of the Schlesinger transformation is algebraically computable from $Y$; see [9, 22]. In fact, the construction problem of Schlesinger transformations is naturally related to rational approximation problems; see also Remark 1.8 .

In this paper we focus on a class of Schlesinger transformations, which is of particular interest from the viewpoint of Hermite's two approximation problems and also of vector continued fractions (see Sect. 3). Note that, for a general Schlesinger transformation other than the present direction, though it can also be controlled by some rational approximation problems but it becomes much more complicated due to the absence of a duality like Mahler's; e.g. $R^{-1}$ seems not to have a concise determinantal representation.

Let us define the $L$-tuple $\boldsymbol{f}={ }^{\mathrm{T}}\left(f_{0}, \ldots, f_{L-1}\right)$ of power series in $w$ as the first column of $\Phi(w)$, where $\Phi(w)$ is the power series part of the solution $Y$ of (1.7) near $z=\infty$. Notice that $f_{0}(0) \neq 0$ certainly holds. Therefore, all the general arguments in Sects.1.1 1.3 are still valid for this specific case, and we are led to the Schlesinger transformation through the two approximation problems for $\boldsymbol{f}$. Now we state the result.

Theorem 1.6. The polynomial matrix $R(z)$ given by (1.5) realizes the Schlesinger transformation $Y \mapsto \hat{Y}=R Y$ shifting the characteristic exponents at $z=\infty$ by $(n(L-1),-n, \ldots,-n)$.

Proof. It follows from the Hermite-Padé approximation condition (1.1) that $R \boldsymbol{f}=O\left(w^{n(L-1)}\right)$. By definition, $R$ takes the form

$$
R=w^{-n}\left(\left[\begin{array}{cccc}
0 & & & \\
* & * & & \\
\vdots & \ddots & \ddots & \\
* & \cdots & * & *
\end{array}\right]+O(w)\right)
$$

Hence, if we write as

$$
R \Phi(w)=\hat{\Phi}(w) \cdot \operatorname{diag}\left(w^{n(L-1)}, w^{-n}, \ldots, w^{-n}\right)
$$


then $\hat{\Phi}$ becomes the same form as $\Phi$. Also, we verify from (1.6) that $R$ does not change the form of the power series expansion of $Y$ near $z=0$.

On the other hand, the coefficient

$$
A(z)=\sum_{i=0}^{N+1} \frac{A_{i}}{z-u_{i}}
$$

of the Fuchsian system (1.7) is transformed as

$$
A \mapsto \hat{A}=R A R^{-1}+\frac{\mathrm{d} R}{\mathrm{~d} z} R^{-1} .
$$

If we remember both $R$ and $R^{-1}$ being polynomials (see Corollary 1.4), then $\hat{A}$ turns out to be a rational function matrix having only simple poles at $S$ as well as the original $A$. In this sense Mahler's duality guarantees the absence of apparent singularities in the new equation $\mathrm{d} \hat{Y} / \mathrm{d} z=\hat{A} \hat{Y}$ satisfied by $\hat{Y}=R Y$.

Remark 1.7. In the rank two case $(L=2)$ a similar construction of Schlesinger transformations as Theorem 1.6 has been established in [16] based on (usual) Padé approximations.

Remark 1.8. A series of pioneering works was done by D. Chudnovsky and G. Chudnovsky on the close connection between rational approximation problems and Riemann's monodromy problem, involving (semi-classical) orthogonal polynomials; see [3, 4] and references therein. The 'Padé method' recently proposed by Yamada [28] is a recipe for Lax formalism of Painlevé equations and, at the same time, for their special solutions, which is based on Padé approximations (or interpolations) of elementary functions; interestingly enough, it is applicable also for various discrete analogues of Painlevé equations beyond the originals; see [7, 17, 19].

The essential idea of the above works could be exemplified by the following: let us consider a function $\varphi(z)=z^{a}(z-1)^{b}(z-u)^{c}$ with $a+b+c=0$. The remainder $\rho:=P \varphi-Q=O\left(z^{-n-1}\right)$ of its Padé approximation then satisfies a second-order linear differential equation denoted by $E$, which may have an apparent singularity besides the four regular singularities $S=\{0,1, u, \infty\} \subset \mathbb{P}^{1}$. However, the two functions $\varphi$ and $\rho$ share the same multi-valuedness since they are rationally related; thus, the monodromy of $E$ is obviously constant with respect to $u$. This fact leads to special solutions of the sixth Painlevé equation $P_{\mathrm{VI}}$, i.e. the isomonodromic deformation (cf. Sect. 4) of a second-order linear differential equation with four regular singularities.

It is interesting to note that such an idea had been recognized implicitly by Laguerre (before the discovery of Painlevé equations); see [13] and also [14].

Remark 1.9. The approximation conditions (1.1) and (1.2) can be interpreted as certain multiorthogonality relations among the $L$-tuples of polynomials $\widetilde{\boldsymbol{Q}}^{(i)}$ and $\widetilde{\boldsymbol{P}}^{(j)}$, respectively; i.e., these polynomials can constitute multi-orthogonal polynomial systems. In this paper, although we do not enter into details on such aspects, we present below the determinantal representations for them, which will crucially work in the last two sections. 


\section{Determinantal representations for approximation polynomi- als and remainders}

In this section we derive determinantal representations for the approximation polynomials $Q_{j}^{(i)}(w)$ and $P_{j}^{(i)}(w)$. We write the power series as

$$
f_{i}(w)=\sum_{j=0}^{\infty} a_{j}^{i} w^{j} \in \mathbb{C} \llbracket w \rrbracket
$$

henceforth; note that the superscript $i$ of $a_{j}^{i}$ is just an index, not an exponent. Introduce the $k \times l$ rectangular Toeplitz matrix

$$
\begin{aligned}
A_{j}^{i}(k, l)= & {\left[a_{j+m-n}^{i}\right]_{1 \leq n \leq l} } \\
& \left.={ }^{1} \begin{array}{cccc}
1 & 2 & & l \\
a_{j}^{i} & a_{j-1}^{i} & \cdots & a_{j-l+1}^{i} \\
a_{j+1}^{i} & a_{j}^{i} & \cdots & a_{j-l+2}^{i} \\
\vdots & \vdots & & \vdots \\
a_{j+k-1}^{i} & a_{j+k-2}^{i} & \cdots & a_{j+k-l}^{i}
\end{array}\right]
\end{aligned}
$$

for the sequence $\left\{a_{j}^{i}\right\}_{j=0}^{\infty}$, where $a_{j}^{i}=0$ if $j<0$. It holds that

$$
\left(A_{n}^{k}(m, n)\right)_{i, j}=\left(A_{m}^{k}(n, m)\right)_{n-j+1, m-i+1}
$$

by definition.

\subsection{Hermite-Padé polynomials}

We can calculate separately for each $i(0 \leq i \leq L-1)$. Therefore, for brevity, we shall express the coefficients of the approximation polynomial $Q_{j}^{(i)}(w)$ as

$$
Q_{j}^{(i)}(w)=\sum_{k=0}^{n-1+\delta_{i, j}} b_{j, k} w^{k}
$$

with omitting the index $i$. The condition (1.1) implies vanishing of the coefficients of $1, w, w^{2}, \ldots, w^{n L-1}$ in the left-hand side. Consequently, we have a system

$$
\left.\mathcal{A}^{(i)}\left[\begin{array}{c}
\boldsymbol{b}_{0} \\
\boldsymbol{b}_{1} \\
\vdots \\
\boldsymbol{b}_{L-1}
\end{array}\right]=\left[\begin{array}{c}
0 \\
0 \\
\vdots \\
0
\end{array}\right]\right\} n L
$$

of homogeneous linear equations for the $n L+1$ unknowns

$$
\boldsymbol{b}_{j}={ }^{\mathrm{T}}\left(b_{j, 0}, \ldots, b_{j, n-1+\delta_{i, j}}\right) \quad(0 \leq j \leq L-1)
$$


where

$$
\mathcal{A}^{(i)}=[\underbrace{A_{0}^{0}(n L, n) \cdots A_{0}^{i-1}(n L, n) A_{0}^{i}(n L, n+1)}_{i+1 \text { blocks }} \underbrace{A_{-1}^{i+1}(n L, n) \cdots A_{-1}^{L-1}(n L, n)}_{L-i-1 \text { blocks }}] .
$$

The solution of (2.4) is unique up to multiplication by constants if and only if the rank of the $n L \times(n L+1)$ matrix $\mathcal{A}^{(i)}$ equals $n L$ (which we will always assume).

Interestingly enough, we have the following determinantal representation of $Q_{j}^{(i)}(w)$.

Proposition 2.1. It holds that

$$
Q_{j}^{(i)}(w)=\frac{1}{\mathrm{NQ}^{(i)}} \operatorname{det}\left[\frac{\mathbf{0} \overbrace{1, w, \ldots, w^{n-1+\delta_{i, j}}}^{\text {th block }} \mathbf{0}}{\mathcal{A}^{(i)}}\right],
$$

where $\mathrm{NQ}^{(i)}$ are some normalizing constants.

Proof. Consider

$$
\rho_{i}(w)=Q_{0}^{(i)} f_{0}+\cdots+Q_{i}^{(i)} f_{i}+w Q_{i+1}^{(i)} f_{i+1}+\cdots+w Q_{L-1}^{(i)} f_{L-1},
$$

which is the remainder of the approximation condition (1.1). Substituting (2.5) shows that

$$
\begin{aligned}
& \rho_{i}(w)=\frac{1}{\mathrm{NQ}^{(i)}} \operatorname{det}\left[\begin{array}{cccc}
\overbrace{f_{0}, f_{0} w, \ldots, f_{0} w^{n-1}}^{\text {0th block }} & \ldots & \overbrace{f_{i-1}, f_{i-1} w, \ldots, f_{i-1} w^{n-1}}^{(i-1) \text { th block }} & \overbrace{f_{i}, f_{i} w, \ldots, f_{i} w^{n}}^{\text {ith block }} \\
A_{0}^{0}(n L, n) & \cdots & A_{0}^{i-1}(n L, n) & A_{0}^{i}(n L, n+1)
\end{array}\right. \\
& \left.\begin{array}{ccc}
\overbrace{f_{i+1} w, f_{i+1} w^{2}, \ldots, f_{i+1} w^{n}}^{(i+1) \text { th block }} & \ldots & \overbrace{f_{L-1} w, f_{L-1} w^{2}, \ldots, f_{L-1} w^{n}}^{(L-1) \text { th block }} \\
A_{-1}^{i+1}(n L, n) & \ldots & A_{-1}^{L-1}(n L, n)
\end{array}\right] .
\end{aligned}
$$

Therefore, if we put $\rho_{i}(w)=\sum_{k=0}^{\infty} \rho_{k}^{i} w^{k}$, then the coefficients read

$$
\rho_{k}^{i}=\frac{1}{\mathrm{NQ}^{(i)}} \operatorname{det}\left[\begin{array}{ccccccc}
A_{k}^{0}(1, n) & \cdots & A_{k}^{i-1}(1, n) & A_{k}^{i}(1, n+1) & A_{k-1}^{i+1}(1, n) & \cdots & A_{k-1}^{L-1}(1, n) \\
A_{0}^{0}(n L, n) & \cdots & A_{0}^{i-1}(n L, n) & A_{0}^{i}(n L, n+1) & A_{-1}^{i+1}(n L, n) & \cdots & A_{-1}^{L-1}(n L, n)
\end{array}\right] .
$$

It is immediate from a property of determinants to verify $\rho_{k}^{i}=0$ for any $k$ less than $n L$; thus, we have $\rho_{i}(w)=O\left(w^{n L}\right)$ indeed.

We will normalize the polynomials so that its diagonal part $Q_{i}^{(i)}(w)$ becomes monic as well as in Sect. 1.3, Accordingly, the normalizing constant $\mathrm{NQ}^{(i)}$ should be

$$
\begin{aligned}
\mathrm{NQ}^{(i)} & =\operatorname{det}\left[\frac{\overbrace{0, \ldots, 0,1}^{i \text { th block }}}{\mathcal{A}^{(i)}}\right] \\
& =(-1)^{n(i+1)} \operatorname{det}\left[\begin{array}{llllll}
A_{0}^{0}(n L, n) & \cdots & A_{0}^{i}(n L, n) & A_{-1}^{i+1}(n L, n) & \cdots & A_{-1}^{L-1}(n L, n)
\end{array}\right] .
\end{aligned}
$$


Thus, the leading coefficient $\rho_{n L}^{i}$ of the remainder is given by

$$
\begin{gathered}
\rho_{n L}^{i}=\frac{(-1)^{n L}}{\mathrm{NQ}^{(i)}} \operatorname{det}\left[\begin{array}{cccc}
A_{0}^{0}(n L+1, n) & \cdots & A_{0}^{i-1}(n L+1, n) & A_{0}^{i}(n L+1, n+1) \\
A_{-1}^{i+1}(n L+1, n) & \cdots & A_{-1}^{L-1}(n L+1, n)
\end{array}\right]
\end{gathered}
$$

the constant term of the polynomial $Q_{i}^{(i)}(w)(i \neq 0)$ is given by

$$
\begin{aligned}
Q_{i}^{(i)}(0) & =\frac{1}{\mathrm{NQ}^{(i)}} \operatorname{det}\left[\begin{array}{llll}
\mathbf{0} \overbrace{1,0, \ldots, 0}^{i \text { th block }} & \mathbf{0} \\
\mathcal{A}^{(i)}
\end{array}\right] \\
& =\frac{(-1)^{n i}}{\mathrm{NQ}^{(i)}} \operatorname{det}\left[\begin{array}{llllll}
A_{0}^{0}(n L, n) & \cdots & A_{0}^{i-1}(n L, n) & A_{-1}^{i}(n L, n) & \cdots & A_{-1}^{L-1}(n L, n)
\end{array}\right] .
\end{aligned}
$$

Remark 2.2. We here restrict ourselves to the case where $f_{0}(w)=1$. Let us introduce the blockToeplitz determinant

$$
\Delta^{(i)}=\operatorname{det}[\underbrace{A_{n}^{1}(m, n) \cdots A_{n}^{i-1}(m, n)}_{i-1 \text { blocks }} \underbrace{A_{n-1}^{i}(m, n) \cdots A_{n-1}^{L-1}(m, n)}_{L-i \text { blocks }}]
$$

of size $m=n(L-1)$ for each $i(1 \leq i \leq L)$; e.g. $\mathrm{NQ}^{(i)}=(-1)^{n(i+1)} \Delta^{(i+1)}$. We see in particular that

$$
\begin{aligned}
\rho_{n L}^{0} & =(-1)^{m} \frac{\Delta^{(L)}}{\Delta^{(1)}}, \\
Q_{i}^{(i)}(0) & =(-1)^{n} \frac{\Delta^{(i)}}{\Delta^{(i+1)}} \quad(1 \leq i \leq L-1) .
\end{aligned}
$$

These simple formulae will be used later in Sect. 5.

\subsection{Simultaneous Padé polynomials}

Suppose $f_{0}(w)=1$ for simplicity. Or, equivalently, we may understand that we have renamed $f_{i} / f_{0}$ $(i \neq 0)$ as $f_{i}$. For a given power series $F(w)=\sum_{k=0}^{\infty} F_{k} w^{k}$, we employ the notation

$$
[F(w)]_{a}^{b}=\sum_{k=a}^{b} F_{k} w^{k}
$$

denoting its section between $w^{a}$ and $w^{b}$ if $a \leq b$. From now on, we set

$$
m=n(L-1)
$$

as well as in Remark 2.2 .

First we shall construct the formulae for $P_{0}^{(j)}(0 \leq j \leq L-1)$. 
(i) Case $j=0 \quad$ The approximation condition (1.2) requires that

$$
\left[f_{i} P_{0}^{(0)}\right]_{m}^{m+n-1}=0 \quad(1 \leq i \leq L-1)
$$

since $P_{i}^{(0)}(i \neq 0)$ is a polynomial of degree at most $m-1$. If we write

$$
P_{0}^{(0)}(w)=\sum_{k=0}^{m} b_{k} w^{k},
$$

then we find a system

$$
\left.\left[\begin{array}{c}
A_{m}^{1}(n, m+1) \\
A_{m}^{2}(n, m+1) \\
\vdots \\
A_{m}^{L-1}(n, m+1)
\end{array}\right]\left[\begin{array}{c}
b_{0} \\
b_{1} \\
\vdots \\
b_{m}
\end{array}\right]=\left[\begin{array}{c}
0 \\
\vdots \\
0
\end{array}\right]\right\} m
$$

of homogeneous linear equations for the $m+1$ unknowns $b_{0}, \ldots, b_{m}$. The solution of (2.6) is unique up to multiplication by constants if and only if the rank of the $m \times(m+1)$ matrix in the left-hand side equals $m$.

(ii) Case $1 \leq j \leq L-1 \quad$ Similarly, it follows from (1.2) that

$$
\begin{aligned}
& {\left[f_{i} P_{0}^{(j)}\right]_{m}^{m+n-1}=0 \quad(1 \leq i \leq j-1)} \\
& {\left[f_{j} P_{0}^{(j)}\right]_{m}^{m+n-2}=0 \quad(i=j)} \\
& {\left[f_{i} P_{0}^{(j)}\right]_{m-1}^{m+n-2}=0 \quad(j+1 \leq i \leq L-1) .}
\end{aligned}
$$

These amount to the simultaneous linear equation

$$
\left.\left[\begin{array}{c}
A_{m}^{1}(n, m) \\
\vdots \\
A_{m}^{j-1}(n, m) \\
A_{m}^{j}(n-1, m) \\
A_{m-1}^{j+1}(n, m) \\
\vdots \\
A_{m-1}^{L-1}(n, m)
\end{array}\right]\left[\begin{array}{c}
b_{0} \\
b_{1} \\
\vdots \\
b_{m-1}
\end{array}\right]=\left[\begin{array}{c}
0 \\
\vdots \\
0
\end{array}\right]\right\} m-1
$$

for the $m$ unknown coefficients $b_{0}, \ldots, b_{m-1}$ of the polynomial

$$
P_{0}^{(j)}(w)=\sum_{k=0}^{m-1} b_{k} w^{k} .
$$

Proposition 2.3. The polynomials $P_{0}^{(j)}$ admit the following determinantal representations:

$$
P_{0}^{(0)}(w)=\frac{1}{\mathrm{NP}^{(0)}} \operatorname{det}\left[\begin{array}{c}
1, w, w^{2}, \ldots, w^{m} \\
A_{m}^{1}(n, m+1) \\
\vdots \\
A_{m}^{L-1}(n, m+1)
\end{array}\right]
$$


and

$$
P_{0}^{(j)}(w)=\frac{1}{\mathrm{NP}^{(j)}} \operatorname{det}\left[\begin{array}{c}
1, w, w^{2}, \ldots, w^{m-1} \\
A_{m}^{1}(n, m) \\
\vdots \\
A_{m}^{j-1}(n, m) \\
A_{m}^{j}(n-1, m) \\
A_{m-1}^{j+1}(n, m) \\
\vdots \\
A_{m-1}^{L-1}(n, m)
\end{array}\right]
$$

for $1 \leq j \leq L-1$, where $\mathrm{NP}^{(j)}$ are some normalizing constants.

Next the other $P_{i}^{(j)}(i \neq 0)$ can be written as follows:

$$
\begin{array}{lll}
\bullet \text { if } j=0 & P_{i}^{(0)}=\left[f_{i} P_{0}^{(0)}\right]_{0}^{m-1} & \\
\text { • if } 1 \leq j \leq L-1 & P_{i}^{(j)}=\left[f_{i} P_{0}^{(j)}\right]_{0}^{m-1} & \text { for } 1 \leq i \leq j-1, \\
& P_{j}^{(j)}=w\left[f_{j} P_{0}^{(j)}\right]_{0}^{m-1} & \text { for } i=j, \\
& P_{i}^{(j)}=w\left[f_{i} P_{0}^{(j)}\right]_{0}^{m-2} & \text { for } j+1 \leq i \leq L-1 .
\end{array}
$$

We will choose the normalization so that the diagonal part $P_{i}^{(i)}(w)$ becomes monic. Accordingly, we obtain

$$
\mathrm{NP}^{(0)}=\operatorname{det}\left[\begin{array}{c}
0, \ldots, 0,1 \\
A_{m}^{1}(n, m+1) \\
\vdots \\
A_{m}^{L-1}(n, m+1)
\end{array}\right]=(-1)^{m} \operatorname{det}\left[\begin{array}{c}
A_{m}^{1}(n, m) \\
\vdots \\
A_{m}^{L-1}(n, m)
\end{array}\right]=(-1)^{\frac{m(m-n)}{2}+n(L-1)} \Delta^{(L)}
$$

and

$$
\mathrm{NP}^{(j)}=\operatorname{det}\left[\begin{array}{c}
a_{m-1}^{j}, a_{m-2}^{j}, \ldots, a_{0}^{j} \\
A_{m}^{1}(n, m) \\
\vdots \\
A_{m}^{j-1}(n, m) \\
A_{m}^{j}(n-1, m) \\
A_{m-1}^{j+1}(n, m) \\
\vdots \\
A_{m-1}^{L-1}(n, m)
\end{array}\right]=(-1)^{n(j-1)} \operatorname{det}\left[\begin{array}{c}
A_{m}^{1}(n, m) \\
\vdots \\
A_{m}^{j-1}(n, m) \\
A_{m-1}^{j}(n, m) \\
\vdots \\
A_{m-1}^{L-1}(n, m)
\end{array}\right]=(-1)^{\frac{m(m-n)}{2}+n(j-1)} \Delta^{(j)}
$$

for $1 \leq j \leq L-1$. Here we have employed the notation of the block-Toeplitz determinant (see Remark 2.2) in view of (2.3).

Remark 2.4. If $i \geq j \geq 1, P_{i}^{(j)}(w)$ is divisible by $w$. By construction (see Corollary 1.4), we observe that $R^{-1}$ takes the form (cf. (1.9))

$$
R^{-1}=w^{-m}\left(\left[\begin{array}{ll}
* & \\
\vdots & 0 \\
* &
\end{array}\right]+O(w)\right) .
$$




\section{From vector continued fractions to Schlesinger transforma- tions}

In this section we present an alternative construction of the same Schlesinger transformation (considered in Sect. 1) through an algorithm for expanding a vector-valued function into a vector continued fraction.

\subsection{Algorithm for vector continued fraction expansion}

Let $\boldsymbol{f}={ }^{\mathrm{T}}\left(f_{0}, \ldots, f_{L-1}\right)$ be the $L$-tuple of formal power series (2.1). For simplicity, we assume tentatively that $f_{i}(0) \neq 0$ for all $i$. We abbreviate the constant term $a_{0}^{i}$ of $f_{i}(w)=\sum_{j=0}^{\infty} a_{j}^{i} w^{j}$ as $a^{i}$.

First we apply a left multiplication of a permutation matrix to $f$ :

$$
\left[\begin{array}{cccc}
0 & & & 1 \\
1 & 0 & & \\
& \ddots & \ddots & \\
& & 1 & 0
\end{array}\right]\left[\begin{array}{c}
f_{0} \\
f_{1} \\
\vdots \\
f_{L-1}
\end{array}\right]=\left[\begin{array}{c}
f_{L-1} \\
f_{0} \\
\vdots \\
f_{L-2}
\end{array}\right] .
$$

Next we eliminate the constant term of $f_{i}$ by a subtraction of constant multiple of $f_{i+1}$ for each $0 \leq i \leq L-2$ and by a multiplication by $w$ for $i=L-1$ :

$$
\left[\begin{array}{cccccc}
w & & & & & \\
& 1 & -\frac{a^{0}}{a^{1}} & & & \\
& & 1 & -\frac{a^{1}}{a^{2}} & & \\
& & & \ddots & \ddots & \\
& & & & 1 & -\frac{a^{L-3}}{a^{L-2}} \\
-\frac{a^{L-2}}{a^{L-1}} & & & & & 1
\end{array}\right]\left[\begin{array}{c}
f_{L-1} \\
f_{0} \\
f_{1} \\
\vdots \\
f_{L-3} \\
f_{L-2}
\end{array}\right]=O(w)=w\left[\begin{array}{c}
f_{0}^{\prime} \\
f_{1}^{\prime} \\
\vdots \\
f_{L-1}^{\prime}
\end{array}\right] .
$$

Eventually we obtain a new $L$-tuple $\boldsymbol{f}^{\prime}={ }^{\mathrm{T}}\left(f_{0}^{\prime}, \ldots, f_{L-1}^{\prime}\right)$ of power series from the original $\boldsymbol{f}$.

The above procedure is summarized as a left multiplication

$$
\boldsymbol{f}^{\prime}=T \boldsymbol{f}
$$

of an invertible matrix

$$
T=\frac{1}{w}\left[\begin{array}{cccccc}
0 & & & & & w \\
1 & -\frac{a^{0}}{a^{1}} & & & & \\
& 1 & -\frac{a^{1}}{a^{2}} & & & \\
& & \ddots & \ddots & & \\
& & & 1 & -\frac{a^{L-3}}{a^{L-2}} & \\
& & & & 1 & -\frac{a^{L-2}}{a^{L-1}}
\end{array}\right]
$$

with $\operatorname{det} T=(-w)^{1-L}$. This is an analogue of the Euclidean algorithm and can be repeated generically. Let $\boldsymbol{f}[k]={ }^{\mathrm{T}}\left(f_{0}[k], \ldots, f_{L-1}[k]\right)$ denote the corresponding vector of power series at the $k$ th step and let $a^{i}[k]$ denote their constant terms. Hence, we have

$$
\boldsymbol{f}[k+1]=T[k] \boldsymbol{f}[k] \text { and } \boldsymbol{f}[0]=\boldsymbol{f},
$$


where

$$
T[k]=\frac{1}{w}\left[\begin{array}{cccccc}
0 & & & & & w \\
1 & -\frac{a^{0}[k]}{a^{1}[k]} & & & & \\
1 & -\frac{a^{1}[k]}{a^{2}[k]} & & & \\
& & \ddots & \ddots & & \\
& & 1 & -\frac{a^{L-3}[k]}{a^{L-2}[k]} & \\
& & & & 1 & -\frac{a^{L-2}[k]}{a^{L-1}[k]}
\end{array}\right] .
$$

On the other hand, solving (3.1) for $f$ yields

$$
f_{i}=w \sum_{j=i}^{L-1} \frac{a^{i}}{a^{j}} f_{j+1}^{\prime} \quad(0 \leq i \leq L-1)
$$

where $f_{L}^{\prime}=f_{0}^{\prime} / w$. Let us introduce the inhomogeneous coordinates $\varphi={ }^{\mathrm{T}}\left(\varphi_{1}, \ldots, \varphi_{L-1}\right)$ by $\varphi_{i}=$ $f_{i} / f_{0}$. Therefore, we have

$$
\frac{\varphi_{i}}{\varphi_{L-1}}=\frac{a^{i}}{a^{L-1}}+w \sum_{j=i}^{L-2} \frac{a^{i}}{a^{j}} \varphi_{j+1}^{\prime} \quad(0 \leq i \leq L-2)
$$

where $\varphi_{0}=1$.

Definition 3.1 (cf. [18, 20]). Let $\varphi={ }^{\mathrm{T}}\left(\varphi_{1}, \ldots, \varphi_{L-1}\right)$ be an $(L-1)$-vector such that $\varphi_{1} \neq 0$. Then the vector

$$
\iota(\varphi)=\frac{1}{\varphi}=\mathrm{T}\left(\frac{\varphi_{2}}{\varphi_{1}}, \frac{\varphi_{3}}{\varphi_{1}}, \ldots, \frac{\varphi_{L-1}}{\varphi_{1}}, \frac{1}{\varphi_{1}}\right)
$$

is called the reciprocal of $\varphi$. Note that $\iota^{L}=\mathrm{id}$.

Under this notation, the correspondence (3.4) can be translated into

$$
\mathrm{T}\left(\frac{1}{\varphi_{L-1}}, \frac{\varphi_{1}}{\varphi_{L-1}}, \ldots, \frac{\varphi_{L-2}}{\varphi_{L-1}}\right)=\iota^{-1}(\varphi)=\boldsymbol{a}+w B \boldsymbol{\varphi}^{\prime}
$$

or equivalently into

$$
\varphi=\frac{1}{\boldsymbol{a}+w B \varphi^{\prime}}
$$

where

$$
\boldsymbol{a}=\frac{1}{a^{L-1}}\left[\begin{array}{c}
a^{0} \\
a^{1} \\
\vdots \\
a^{L-2}
\end{array}\right] \quad \text { and } \quad B=\left[\begin{array}{ccccc}
1 & \frac{a^{0}}{a^{1}} & \frac{a^{0}}{a^{2}} & \cdots & \frac{a^{0}}{a^{L-2}} \\
& 1 & \frac{a^{1}}{a^{2}} & \cdots & \frac{a^{1}}{a^{L-2}} \\
& \ddots & \ddots & \vdots \\
& & & 1 & \frac{a^{L-3}}{a^{L-2}} \\
& & & 1
\end{array}\right]
$$

Namely, the vector $\boldsymbol{a}$ is determined as the constant term of $\iota^{-1}(\varphi)$ and the matrix $B$ is then specified by $\boldsymbol{a}$. Let $\boldsymbol{\varphi}[k]$ denote the inhomogeneous coordinates of the vector $\boldsymbol{f}[k] \in \mathbb{C}^{L} \llbracket w \rrbracket$ at the $k$ th step. 
Taking the reciprocal repeatedly in this way, we obtain formally the vector continued fraction

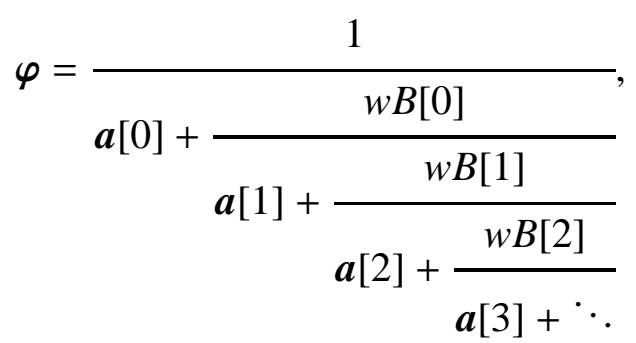

which is regarded as an $(L-1)$-dimensional generalization of the Stieltjes-type continued fraction. Refer to [11, Appendix A] for a classification of continued fractions. Our algorithm differs from the other known examples such as the Jacobi-Perron algorithm; cf. [20, 21]. Note also that some dynamical system, like the Toda lattice, has been studied based on the connection among the Jacobi-Perron algorithm, rational approximations and bi-orthogonal polynomials; see [12].

The following theorem can be verified straightforwardly through the above algorithm, as well as the case of a Stieltjes-type continued fraction (i.e., $L=2$ case).

Theorem 3.2. The kth convergents (rational functions)

$$
\begin{aligned}
& \boldsymbol{\Pi}_{1}=\frac{1}{\boldsymbol{a}[0]}, \\
& \boldsymbol{\Pi}_{k}=\frac{1}{\boldsymbol{a}[0]+\frac{w B[0]}{\boldsymbol{a}[1]+\frac{w B[1]}{\boldsymbol{a}[2]+\ddots+\frac{w B[k-2]}{\boldsymbol{a}[k-1]}}}} \in \mathbb{C}^{L-1}(w) \quad(k \geq 2)
\end{aligned}
$$

of the vector continued fraction (3.5) provide approximants of the vector $\varphi \in \mathbb{C}^{L-1} \llbracket w \rrbracket$ of power series in the sense that $\boldsymbol{\varphi}-\boldsymbol{\Pi}_{k}=O\left(w^{k}\right)$.

In calculating $\boldsymbol{\Pi}_{k}$, it is convenient to apply the projective transformations (3.3) successively as follows:

$$
\left[\begin{array}{c}
\varpi_{0} \\
\varpi_{1} \\
\vdots \\
\varpi_{L-1}
\end{array}\right]=T[0]^{-1} T[1]^{-1} \cdots T[k-1]^{-1}\left[\begin{array}{c}
1 \\
0 \\
\vdots \\
0
\end{array}\right] \quad \text { and } \quad \boldsymbol{\Pi}_{k}=\frac{1}{\varpi_{0}}\left[\begin{array}{c}
\varpi_{1} \\
\varpi_{2} \\
\vdots \\
\varpi_{L-1}
\end{array}\right]
$$

\subsection{Schlesinger transformations, revisited}

Let $Y=Y(z)$ be a solution (1.8) of the Fuchsian system (1.7) having the local behaviors

$$
\begin{aligned}
Y & =\Psi(z) \cdot \operatorname{diag}\left(z^{\varepsilon_{0, j}}\right)_{0 \leq j \leq L-1} \quad(\text { near } z=0) \\
& =\Phi(w) \cdot \operatorname{diag}\left(w^{\varepsilon_{\infty, j}}\right)_{0 \leq j \leq L-1} \cdot C \quad(\text { near } z=1 / w=\infty)
\end{aligned}
$$


where the power series parts are normalized by

$$
\Psi(z)=\left[\begin{array}{ccc}
1 & \cdots & * \\
& \ddots & \vdots \\
& & 1
\end{array}\right]+O(z), \quad \Phi(w)=\left[\begin{array}{ccc}
* & \\
\vdots & \ddots & \\
* & \cdots & *
\end{array}\right]+O(w)
$$

and $C$ is the connection matrix. Let $\boldsymbol{f}={ }^{\mathrm{T}}\left(f_{0}, \ldots, f_{L-1}\right)$ denote the first column of $\Phi(w)$.

It is clear from the construction of the matrix $T=T[0]$ that

$$
T \Phi=\left(\left[\begin{array}{ccc}
* & & \\
\vdots & \ddots & \\
* & \cdots & *
\end{array}\right]+O(w)\right) \cdot \operatorname{diag}\left(1, w^{-1}, \ldots, w^{-1}\right)
$$

near $w=0(z=\infty)$. On the other hand, it holds that

$$
\begin{aligned}
T \Psi & =z\left(\left[\begin{array}{ccccc}
* & * & \cdots & * & 1 \\
1 & * & \cdots & * & 0 \\
& 1 & \ddots & \vdots & \vdots \\
& & \ddots & * & 0 \\
& & & 1 & 0
\end{array}\right]+O(z)\right] \cdot \operatorname{diag}\left(1, \ldots, 1, z^{-1}\right) \\
& =z\left(\left[\begin{array}{ccc}
1 & \cdots & * \\
& \ddots & \vdots \\
& & 1
\end{array}\right]+O(z)\right]\left[\begin{array}{cccc}
0 & & & z^{-1} \\
1 & 0 & & \\
& \ddots & \ddots & \\
& & 1 & 0
\end{array}\right]
\end{aligned}
$$

near $z=0$. After we repeat the same procedure $L$ times, the power series part thus recovers its original form:

$$
T[L-1] \cdots T[1] T[0] \Psi=z^{L-1}\left(\left[\begin{array}{ccc}
1 & \cdots & * \\
& \ddots & \vdots \\
& & 1
\end{array}\right]+O(z)\right) .
$$

In conclusion, the matrix $z^{1-L} T[L-1] \cdots T[1] T[0]$ turns out to be a polynomial in $z$ and to be the multiplier of the Schlesinger transformation shifting the characteristic exponents at $z=\infty$ by $(L-1,-1, \ldots,-1)$; cf. Theorem 1.6 .

Remark 3.3. In fact, the same approximation problem considered in Sect. 1 appears in the following manner. Concerning the polynomial matrix $w^{L} T[L-1] \cdots T[1] T[0]$, we observe from the form of $T[k]$ that the diagonal entries are all monic linear functions, the strictly upper triangular part is linear and divisible by $w$, and the strictly lower triangular part is a constant. Moreover, in view of (3.2) we have

$$
w^{L} T[L-1] \cdots T[1] T[0] f=O\left(w^{L}\right),
$$

which coincides with the approximation condition (1.1), and thus

$$
w^{L} T[L-1] \cdots T[1] T[0]=\left[\begin{array}{c}
\widetilde{\boldsymbol{Q}}^{(0)}(w) \\
\vdots \\
\widetilde{\boldsymbol{Q}}^{(L-1)}(w)
\end{array}\right]
$$

under $n=1$. 


\section{Application to isomonodromic deformations}

In this section we first review some basic results on the Schlesinger system, which governs isomonodromic deformations of a Fuchsian system of linear ordinary differential equations. As explained in Sect. 1.4, a Schlesinger transformation preserves the monodromy of the Fuchsian system under consideration and, thereby, leads to a discrete symmetry of the associated Schlesinger system. Combining this fact with the result in Sect. 2 reveals a determinantal nature of isomonodromic deformations. Next we treat a particular case of the Schlesinger systems unifying various Painlevétype differential equations and show its relationship with certain hypergeometric functions, which will be needed later.

\subsection{Schlesinger systems and their symmetries}

Let us consider again the $L \times L$ Fuchsian system (1.7):

$$
\frac{\mathrm{d} Y}{\mathrm{~d} z}=A Y=\sum_{i=0}^{N+1} \frac{A_{i}}{z-u_{i}} Y,
$$

where $u_{0}=1$ and $u_{N+1}=0$. We start with a well-known result on isomonodromic deformations of (4.1).

Theorem 4.1. The monodromy of a fundamental solution $Y$, i.e. $\operatorname{det} Y \neq 0$, does not depend on $\boldsymbol{u}=\left(u_{1}, \ldots, u_{N}\right)$ if and only if

$$
B_{i}=\frac{\partial Y}{\partial u_{i}} Y^{-1} \quad(1 \leq i \leq N)
$$

are rational functions in $z$.

We henceforth impose on our Fuchsian system (4.1) the following assumptions:

(i) all the residue matrices $A_{i}$ are semi-simple, i.e. diagonalizable;

(ii) there is no integer difference other than zero among the eigenvalues of each $A_{i}$.

Let us choose a normalization as before such that $A_{N+1}$ and $A_{N+2}=-\sum_{i=0}^{N+1} A_{i}$ are upper and lower triangular matrices, respectively. Then we can take a fundamental solution $Y=Y(z)$ of the form

$$
\begin{aligned}
Y & =\Psi(z) \cdot \operatorname{diag}\left(z^{\varepsilon_{0, j}}\right)_{0 \leq j \leq L-1} \quad(\text { near } z=0) \\
& =\Phi(w) \cdot \operatorname{diag}\left(w^{\varepsilon_{\infty, j}}\right)_{0 \leq j \leq L-1} \cdot C \quad(\text { near } z=1 / w=\infty)
\end{aligned}
$$

with

$$
\begin{aligned}
& \Psi(z)=\left[\begin{array}{lll}
1 & \cdots & * \\
& \ddots & \vdots \\
& & 1
\end{array}\right]+O(z) \\
& \Phi(w)=\left[\begin{array}{ccc}
* & & \\
\vdots & \ddots & \\
* & \cdots & *
\end{array}\right]+O(w)=\left(\left[\begin{array}{ccc}
1 & \\
\vdots & \ddots & \\
* & \cdots & 1
\end{array}\right]+O(w)\right) \Xi
\end{aligned}
$$


and $\Xi=\operatorname{diag}\left(\xi_{0}, \ldots, \xi_{L-1}\right)$, where each $\xi_{j} \neq 0$ may depend on $\boldsymbol{u}=\left(u_{1}, \ldots, u_{N}\right)$. Moreover, we have

$$
Y=G_{i}\left(I+O\left(z-u_{i}\right)\right)\left(z-u_{i}\right)^{\Lambda_{i}}
$$

near each of the other regular singularities $z=u_{i}(0 \leq i \leq N)$, where $G_{i}$ and $\Lambda_{i}$ are certain constant matrices satisfying $G_{i} \Lambda_{i} G_{i}^{-1}=A_{i}$. Therefore, the monodromy matrices of $Y$ attached to loops around $z=u_{i}(0 \leq i \leq N)$ and $z=0, \infty$ read

$$
e^{2 \pi \sqrt{-1} \Lambda_{i}}, \quad e^{2 \pi \sqrt{-1} \operatorname{diag}\left(\varepsilon_{0, j}\right)_{0 \leq j \leq L-1}}, \quad C^{-1} e^{2 \pi \sqrt{-1} \operatorname{diag}\left(\varepsilon_{\infty, j}\right)_{0 \leq j \leq L-1}} C .
$$

Suppose now that every monodromy matrix of $Y$ is constant with respect to $\boldsymbol{u}$ and, additionally, so is the connection matrix $C$. Then the rational functions $B_{i}=B_{i}(z)$ can be explicitly written as

$$
B_{i}=\frac{A_{i}}{u_{i}-z}-\frac{1}{u_{i}}\left(A_{i}\right)_{\mathrm{LT}},
$$

where $\left(A_{i}\right)_{\mathrm{LT}}$ denotes the lower triangular part of $A_{i}$; see Appendix for details. Note in particular that the diagonal part $\left(A_{i}\right)_{\mathrm{D}}$ of $A_{i}$ is expressible in terms of $\Xi$ as

$$
\left(A_{i}\right)_{\mathrm{D}}=-u_{i} \frac{\partial}{\partial u_{i}} \log \Xi
$$

The compatibility condition

$$
\frac{\partial A}{\partial u_{i}}-\frac{\partial B_{i}}{\partial z}+\left[A, B_{i}\right]=0
$$

of (4.1) and (4.2) is equivalent to a set of nonlinear differential equations for the matrices $A_{i}$ with respect to $\boldsymbol{u}$, which is called the Schlesinger system [22]. If $(L, N)=(2,1)$, then the Schlesinger system reduces to the sixth Painlevé equation $P_{\mathrm{VI}}$.

Next we shall investigate how the solution $Y$ and the coefficient $A=A(z)$ of the Fuchsian system (4.1) are connected with each other. Concerning the power series expansion

$$
Y=\Phi(w) \cdot \operatorname{diag}\left(w^{\varepsilon_{\infty, j}}\right) \cdot C, \quad \Phi(w)=\sum_{k=0}^{\infty} \Phi_{k} w^{k}
$$

at the point of infinity $(z=1 / w=\infty)$, the coefficients $\Phi_{k}$ turn out to be polynomials in the off-diagonal entries of the lower triangular matrix $A_{N+2}=-\sum_{i=0}^{N+1} A_{i}$ through Frobenius' method. Conversely, substituting this solution $Y$ in (4.1), we find that

$$
\begin{aligned}
& z \frac{\mathrm{d} Y}{\mathrm{~d} z}=-w \frac{\mathrm{d} Y}{\mathrm{~d} w} \\
&=-w \frac{\mathrm{d}}{\mathrm{d} w}\left(\Phi_{0}+\Phi_{1} w+\Phi_{2} w^{2}+\cdots\right) \operatorname{diag}\left(w^{\varepsilon_{\infty, j}}\right) \cdot C \\
&=-\left[\left(\Phi_{0}+\Phi_{1} w+\Phi_{2} w^{2}+\cdots\right) \operatorname{diag}\left(\varepsilon_{\infty, j}\right)\right. \\
&\left.+\Phi_{1} w+2 \Phi_{2} w^{2}+3 \Phi_{3} w^{3}+\cdots\right] \operatorname{diag}\left(w^{\varepsilon_{\infty, j}}\right) \cdot C
\end{aligned}
$$

and

$$
\begin{aligned}
z \sum_{i=0}^{N+1} \frac{A_{i}}{z-u_{i}} Y & =\sum_{i=0}^{N+1} \frac{A_{i}}{1-u_{i} w} Y \\
& =\left[A_{N+1}+\sum_{i=0}^{N}\left(1+u_{i} w+u_{i}{ }^{2} w^{2}+\cdots\right) A_{i}\right] Y .
\end{aligned}
$$


Recall $u_{N+1}=0$ here. Equating the coefficients of $w^{k}$ in these power series yields

$$
A_{N+2}=\Phi_{0} \cdot \operatorname{diag}\left(\varepsilon_{\infty, j}\right) \cdot \Phi_{0}^{-1} \quad \text { for } k=0
$$

thus $A_{N+2}$ becomes a polynomial in the entries of the leading coefficient $\Phi_{0}$ of the solution, and also

$$
\sum_{i=0}^{N} u_{i} A_{i}=\left(-\Phi_{1} \cdot \operatorname{diag}\left(\varepsilon_{\infty, j}+1\right)+\Phi_{0} \cdot \operatorname{diag}\left(\varepsilon_{\infty, j}\right) \cdot \Phi_{0}^{-1} \Phi_{1}\right) \Phi_{0}^{-1} \quad \text { for } k=1 .
$$

Moreover, if one needs similar expressions for all other residue matrices $A_{i}$ besides $A_{N+2}$, it is convenient to use the deformation equation (4.2); one can verify in fact

$$
A_{i}=\left(\frac{\partial \Phi_{0}}{\partial u_{i}} \Phi_{0}^{-1} \Phi_{1}-\frac{\partial \Phi_{1}}{\partial u_{i}}\right) \Phi_{0}^{-1} \quad(1 \leq i \leq N) .
$$

In summary, each residue matrix $A_{i}$ of $A(z)$ is expressible as a polynomial in the entries of the coefficients of $Y$ (and their derivatives with respect to $\boldsymbol{u}$ ), and vice versa.

A Schlesinger transformation keeps the monodromy of the Fuchsian system invariant but shifts its characteristic exponents by integers; recall Sect. 1.4. Consequently, it gives rise to a discrete symmetry of the Schlesinger system via the above correspondence between the solutions and coefficients of the Fuchsian system. On the other hand, any ingredient of Schlesinger transformations or of the associated rational approximations is described in terms of block-Toeplitz determinants; recall Sect. 2. This fact thus provides a natural explanation for the determinantal structure appearing in solutions of isomonodromic deformations, e.g. Painlevé equations. Refer to [8] for a detailed investigation of the determinantal structure in Jimbo-Miwa-Ueno's $\tau$-functions [10] for a general framework admitting irregular singularities.

\subsection{Polynomial Hamiltonian system $\mathcal{H}_{L, N}$ of isomonodromy type}

We turn now to a particular case of the Schlesinger systems, which will be the main subject in the rest of this paper.

Consider an $L \times L$ Fuchsian system of the form (4.1) whose spectral type is given by the partitions of $L$ :

$$
\begin{aligned}
& 1, L-1 \quad \text { at } z=u_{i}(0 \leq i \leq N) \text { and } \\
& \underbrace{1,1, \ldots, 1}_{L} \text { at } z=0, \infty,
\end{aligned}
$$

which indicate how the characteristic exponents overlap at each of the $N+3$ singularities. Fix the characteristic exponents as listed in the following table (Riemann scheme):

\begin{tabular}{|c|c|}
\hline Singularity & Characteristic exponents \\
\hline$u_{i}(0 \leq i \leq N)$ & $\left(-\theta_{i}, 0, \ldots, 0\right)$ \\
\hline$u_{N+1}=0$ & $\left(e_{0}, e_{1}, \ldots, e_{L-1}\right)$ \\
\hline$u_{N+2}=\infty$ & $\left(\kappa_{0}-e_{0}, \kappa_{1}-e_{1}, \ldots, \kappa_{L-1}-e_{L-1}\right)$ \\
\hline
\end{tabular}

Assume the sum of all the characteristic exponents equals zero (Fuchs relation), i.e.

$$
\sum_{l=0}^{L-1} \kappa_{l}=\sum_{i=0}^{N} \theta_{i}
$$


Let $A_{N+1}$ and $A_{N+2}=-\sum_{i=0}^{N+1} A_{i}$ be upper and lower triangular matrices, respectively. Then such a Fuchsian system, denoted by $\mathcal{L}_{L, N}$, can be parametrized as follows:

$$
\begin{aligned}
A_{i} & ={ }^{\mathrm{T}}\left(b_{0}^{(i)}, b_{1}^{(i)}, \ldots, b_{L-1}^{(i)}\right) \cdot\left(c_{0}^{(i)}, c_{1}^{(i)}, \ldots, c_{L-1}^{(i)}\right) \quad \text { with } \quad c_{0}^{(i)}=1 \quad(0 \leq i \leq N), \\
A_{N+1} & =\left[\begin{array}{cccc}
e_{0} & w_{0,1} & \cdots & w_{0, L-1} \\
& e_{1} & \ddots & \vdots \\
& \ddots & w_{L-2, L-1} \\
& & e_{L-1}
\end{array}\right],
\end{aligned}
$$

under the relations

$$
\left(\operatorname{tr} A_{i}=\right)-\theta_{i}=\sum_{k=0}^{L-1} b_{k}^{(i)} c_{k}^{(i)}, \quad \kappa_{l}=-\sum_{i=0}^{N} b_{l}^{(i)} c_{l}^{(i)} \quad \text { and } \quad w_{k, l}=-\sum_{i=0}^{N} b_{k}^{(i)} c_{l}^{(i)} \quad(k<l) ;
$$

the last two of which come from the triangularity of $A_{N+2}$. Also, we can and will normalize the characteristic exponents at $z=0$ by

$$
\operatorname{tr} A_{N+1}=\sum_{k=0}^{L-1} e_{k}=\frac{L-1}{2}
$$

without loss of generality.

As shown in [26], the Schlesinger system governing isomonodromic deformations of $\mathcal{L}_{L, N}$ reduces to the multi-time Hamiltonian system $\mathcal{H}_{L, N}$ :

$$
\frac{\partial q_{k}^{(i)}}{\partial x_{j}}=\frac{\partial H_{j}}{\partial p_{k}^{(i)}}, \quad \frac{\partial p_{k}^{(i)}}{\partial x_{j}}=-\frac{\partial H_{j}}{\partial q_{k}^{(i)}} \quad\left(\begin{array}{c}
1 \leq i, j \leq N \\
1 \leq k \leq L-1
\end{array}\right)
$$

Here we let $x_{i}=1 / u_{i}$ and define the Hamiltonian function $H_{i}$ by

$$
x_{i} H_{i}=\sum_{k=0}^{L-1} e_{k} q_{k}^{(i)} p_{k}^{(i)}+\sum_{j=0}^{N} \sum_{0 \leq k<l \leq L-1} q_{k}^{(i)} p_{k}^{(j)} q_{l}^{(j)} p_{l}^{(i)}+\sum_{\substack{j=0 \\ j \neq i}}^{N} \frac{x_{j}}{x_{i}-x_{j}} \sum_{k, l=0}^{L-1} q_{k}^{(i)} p_{k}^{(j)} q_{l}^{(j)} p_{l}^{(i)}
$$

with $x_{0}=q_{l}^{(0)}=q_{0}^{(i)}=1, p_{l}^{(0)}=\kappa_{l}-\sum_{i=1}^{N} q_{l}^{(i)} p_{l}^{(i)}$ and $p_{0}^{(i)}=\theta_{i}-\sum_{k=1}^{L-1} q_{k}^{(i)} p_{k}^{(i)}$. Therefore, $H_{i}$ is a polynomial in the unknowns (canonical variables)

$$
q_{k}^{(i)}=\frac{c_{k}^{(i)}}{c_{k}^{(0)}} \quad \text { and } \quad p_{k}^{(i)}=-b_{k}^{(i)} c_{k}^{(0)} \quad\left(\begin{array}{c}
1 \leq i \leq N \\
1 \leq k \leq L-1
\end{array}\right) .
$$

The number of the constant parameters

$$
(\boldsymbol{e}, \boldsymbol{\kappa}, \boldsymbol{\theta})=\left(e_{0}, \ldots, e_{L-1}, \kappa_{0}, \ldots, \kappa_{L-1}, \theta_{0}, \ldots, \theta_{N}\right)
$$

contained in $\mathcal{H}_{L, N}$ is essentially $2 L+N-1$ in view of (4.7) and (4.9). For example, the case where $L=2$ and any $N \geq 1$ coincides with the Garnier system in $N$-variables and, thereby, the first nontrivial case $\mathcal{H}_{2,1}$ does with the Hamiltonian form of $P_{\mathrm{VI}}$.

Remark 4.2. We have a priori known from their spectral type that the Fuchsian systems equipped with the Riemann scheme (4.6) constitute a $2 N(L-1)$-dimensional family. The coordinates of such a family are called accessory parameters, which are realized by the $2 N(L-1)$ canonical variables (4.10) in this instance. 


\subsection{Solution of $\mathcal{H}_{L, N}$ in terms of hypergeometric function $F_{L, N}$}

Although the phase space of $\mathcal{H}_{L, N}$ is a quite-complicated algebraic variety in general, there exists a family of solutions parametrized by a point in the projective space $\mathbb{P}^{N(L-1)}$ when the constants $(\boldsymbol{e}, \boldsymbol{\kappa}, \boldsymbol{\theta})$ take certain special values. In fact, these solutions are written in terms of the hypergeometric function

$$
F_{L, N}\left[\begin{array}{c}
\boldsymbol{\alpha}, \boldsymbol{\beta} \\
\boldsymbol{\gamma}
\end{array} ; \boldsymbol{x}\right]=\sum_{m_{i} \geq 0} \frac{\left(\alpha_{1}\right)_{|\boldsymbol{m}|} \cdots\left(\alpha_{L-1}\right)_{|\boldsymbol{m}|}\left(\beta_{1}\right)_{m_{1}} \cdots\left(\beta_{N}\right)_{m_{N}}}{\left(\gamma_{1}\right)_{|\boldsymbol{m}|} \cdots\left(\gamma_{L-1}\right)_{|\boldsymbol{m}|}} \frac{x_{1}{ }^{m_{1}} \cdots x_{N}{ }^{m_{N}}}{m_{1} ! \cdots m_{N} !}
$$

where $|\boldsymbol{m}|=m_{1}+\cdots+m_{N}$ and $(a)_{k}=\Gamma(a+k) / \Gamma(a)$. If $(L, N)=(2,1)$, then (4.12) is exactly Gauß's hypergeometric function.

To state the result precisely, we introduce the integral representation

$$
y_{0}=\int_{c} \frac{U(\boldsymbol{t}) \mathrm{d} t_{1} \cdots \mathrm{d} t_{L-1}}{\prod_{l=1}^{L-1}\left(t_{l-1}-t_{l}\right)}=\prod_{l=1}^{L-1} \frac{\Gamma\left(\alpha_{l}\right) \Gamma\left(\gamma_{l}-\alpha_{l}\right)}{\Gamma\left(\gamma_{l}\right)} \times F_{L, N}\left[\begin{array}{c}
\boldsymbol{\alpha}, \boldsymbol{\beta} \\
\boldsymbol{\gamma}
\end{array} ; \boldsymbol{x}\right]
$$

of $F_{L, N}$, where the multi-valued function $U=U(\boldsymbol{t})$ in $\boldsymbol{t}=\left(t_{1}, t_{2}, \ldots, t_{L-1}\right)$ is given by

$$
U(\boldsymbol{t})=\prod_{l=1}^{L-1} t_{l}^{\alpha_{l}-\gamma_{l+1}}\left(t_{l-1}-t_{l}\right)^{\gamma_{l}-\alpha_{l}} \prod_{i=1}^{N}\left(1-x_{i} t_{L-1}\right)^{-\beta_{i}} \quad \text { and } \quad t_{0}=\gamma_{L}=1,
$$

and the cycle $c$ is chosen to be an $(L-1)$-simplex

$$
\left\{0 \leq t_{L-1} \leq \cdots \leq t_{2} \leq t_{1} \leq 1\right\} \subset \mathbb{R}^{L-1} \text {. }
$$

Also, we introduce supplementarily the integrals

$$
y_{k}^{(i)}=\int_{c} \frac{U(\boldsymbol{t}) \mathrm{d} t_{1} \cdots \mathrm{d} t_{L-1}}{\left(x_{i} t_{L-1}-1\right) \prod_{\substack{l=1 \\
l \neq k}}^{L-1}\left(t_{l-1}-t_{l}\right)} \quad\left(\begin{array}{c}
1 \leq i \leq N \\
1 \leq k \leq L-1
\end{array}\right) .
$$

We are now ready to state the hypergeometric solution of $\mathcal{H}_{L, N}$; see [25, Theorem 3.2].

Theorem 4.3. If $\kappa_{0}-\sum_{i=1}^{N} \theta_{i}=0$ then the Hamiltonian system $\mathcal{H}_{L, N}$ possesses a solution

$$
q_{k}^{(i)}=0 \quad \text { and } \quad p_{k}^{(i)}=\theta_{i} \frac{y_{k}^{(i)}}{y_{0}}
$$

under the correspondence

$$
\alpha_{k}=e_{k}-e_{0}, \quad \beta_{i}=-\theta_{i}, \quad \gamma_{k}=e_{k}-e_{0}-\kappa_{k}
$$

of constant parameters.

The vector-valued function

$$
\boldsymbol{y}={ }^{\mathrm{T}}\left(y_{0}, y_{1}^{(1)}, \ldots, y_{L-1}^{(1)}, y_{1}^{(2)}, \ldots, y_{L-1}^{(2)}, \ldots, y_{1}^{(N)}, \ldots, y_{L-1}^{(N)}\right)
$$


satisfies a certain linear Pfaffian system $\mathcal{P}_{L, N}$ of $\operatorname{rank} N(L-1)+1$, whose fundamental solution is prepared by collecting admissible cycles along with the foregoing $(L-1)$-simplex (4.14). Note that the linear space of these cycles, i.e. twisted de Rham homology group, is generated by the chambers framed by the real section of branch locus of $U=U(\boldsymbol{t})$; see [1, 25]. Of course, Theorem 4.3] is valid for any solution $\left\{y_{0}, y_{k}^{(i)}\right\}$ of $\mathcal{P}_{L, N}$.

The Fuchsian system $\mathcal{L}_{L, N}$ is specialized as $\kappa_{0}-\sum_{i=1}^{N} \theta_{i}=0$ and

$$
\begin{aligned}
& b_{0}^{(0)}=0, \quad b_{k}^{(0)}=\kappa_{k} y_{0}, \quad b_{0}^{(i)}=-\theta_{i}, \quad b_{k}^{(i)}=\theta_{i} y_{k}^{(i)}, \\
& c_{k}^{(0)}=\frac{-1}{y_{0}}, \quad c_{k}^{(i)}=0 \quad\left(\begin{array}{c}
1 \leq i \leq N \\
1 \leq k \leq L-1
\end{array}\right)
\end{aligned}
$$

along the above hypergeometric solution of $\mathcal{H}_{L, N}$; it thus becomes reducible. In fact, via the gauge transformation

$$
Y=\left(w^{K_{0}-e_{0}} \prod_{i=1}^{N}\left(1-u_{i} w\right)^{-\theta_{i}} u_{i}^{\theta_{i}}\right) Y^{\prime},
$$

we have a solution of the form

$$
\begin{aligned}
& Y^{\prime}=\left[\begin{array}{cccc}
1 & 0 & \cdots & 0 \\
f_{1} & & & \\
\vdots & & W & \\
f_{L-1} & & &
\end{array}\right] \\
& =\left(\left[\begin{array}{ccc}
1 & & \\
\vdots & \ddots & \\
* & \cdots & 1
\end{array}\right]+O(w)\right) \cdot \operatorname{diag}\left(w^{K_{j}-e_{j}-\kappa_{0}+e_{0}}\right)_{0 \leq j \leq L-1},
\end{aligned}
$$

where $f_{k}=f_{k}(w)(1 \leq k \leq L-1)$ are holomorphic functions at $w=0$ defined by the integrals

$$
f_{k}=\int_{c} \frac{U(\boldsymbol{t}) \mathrm{d} t_{1} \cdots \mathrm{d} t_{L-1}}{\left(1-w t_{L-1}\right) \prod_{\substack{l=1 \\ l \neq k}}^{L-1}\left(t_{l-1}-t_{l}\right)} .
$$

Note that an $(L-1) \times(L-1)$ matrix $W$ can be described by Thomae's hypergeometric function $F_{L-1,1}$. For details we refer to [27], in which a curious coincidence between $\mathcal{P}_{L, N+1}$ and the $\operatorname{Lax}$ pair of $\mathcal{H}_{L, N}$, i.e. the pair of the original Fuchsian system (4.1) and its deformation equation (4.2), is also discussed.

Our aim here is to generalize Theorem 4.3 by application of Schlesinger transformations starting from this hypergeometric solution at $\kappa_{0}-\sum_{i=1}^{N} \theta_{i}=0$. Notice that the Schlesinger transformation established in Theorem 1.6 shifts the constant parameters (4.11) as

$$
\left(\kappa_{0}, \kappa_{1}, \ldots, \kappa_{L-1}\right) \mapsto\left(\kappa_{0}+n(L-1), \kappa_{1}-n, \ldots, \kappa_{L-1}-n\right)
$$

while all the others are unchanged; cf. the Riemann scheme (4.6). Hence, by virtue of the algebraic relation between the solution of $\mathcal{L}_{L, N}$ and the canonical variables of $\mathcal{H}_{L, N}$ (recall Sect. 4.1 and also (4.8) and (4.10) $)$, we know in principle how to derive a solution $\left(\hat{q}_{k}^{(i)}, \hat{p}_{k}^{(i)}\right)$ of $\mathcal{H}_{L, N}$ at $\kappa_{0}-\sum_{i=1}^{N} \theta_{i}=$ $n(L-1)$ for any positive integer $n$ even though the resulting expression in this way will be terribly complicated. In the next section we explore this problem to achieve much simpler formulae for these special solutions. 


\section{Solutions of $\mathcal{H}_{L, N}$ in terms of iterated hypergeometric inte- grals}

This section is concerned with the Schlesinger transform of the hypergeometric solution of $\mathcal{H}_{L, N}$. We present its explicit formula by using the block-Toeplitz determinant whose entries are given by the hypergeometric functions. Key ingredients of the argument are the determinantal representations for the approximation polynomials; see Sect. 2. Moreover, we prove through Fubini's theorem and the Vandermonde determinant that these block-Toeplitz determinants can be written in the form of iterated hypergeometric integrals. Our result will be summarized in Theorem 5.3, which is regarded as a generalization of Theorem 4.3, i.e. the previously known hypergeometric solution of $\mathcal{H}_{L, N}$.

\subsection{Preliminaries}

Let $f_{0}(w), f_{1}(w), \ldots, f_{L-1}(w)$ be the functions defined by

$$
\begin{aligned}
& f_{0}(w) \equiv 1, \\
& f_{k}(w)=\int_{c} \frac{U(\boldsymbol{t}) \mathrm{d} t_{1} \cdots \mathrm{d} t_{L-1}}{\left(1-w t_{L-1}\right) \prod_{\substack{l=1 \\
l \neq k}}^{L-1}\left(t_{l-1}-t_{l}\right)} \quad(1 \leq k \leq L-1) .
\end{aligned}
$$

If the cycle $c$ is chosen such that $\left|t_{L-1}\right|<\infty$, then $f_{k}(w)$ is holomorphic at $w=0$. For instance, it is enough to choose a bounded cycle as $c$. Accordingly, we have a power series expansion

$$
f_{k}(w)=\sum_{j=0}^{\infty} h_{j}^{k} w^{j}=h_{0}^{k}+h_{1}^{k} w+h_{2}^{k} w^{2}+\cdots
$$

with the coefficients

$$
h_{j}^{k}=\int_{c} \frac{t_{L-1}^{j} U(\boldsymbol{t}) \mathrm{d} t_{1} \cdots \mathrm{d} t_{L-1}}{\prod_{\substack{l=1 \\ l \neq k}}^{L-1}\left(t_{l-1}-t_{l}\right)}
$$

for $1 \leq k \leq L-1$. Observe that each $h_{j}^{k}$ can be regarded as a moment

$$
h_{j}^{k}=\int_{\operatorname{pr}(c)} s^{j} \mathrm{~d} \mu_{k}(s)
$$

of the 'measure'

$$
\mathrm{d} \mu_{k}\left(t_{L-1}\right)=\left(\int_{c \mid t_{L-1}} \frac{U(\boldsymbol{t}) \mathrm{d} t_{1} \cdots \mathrm{d} t_{L-2}}{\prod_{\substack{l=1 \\ l \neq k}}^{L-1}\left(t_{l-1}-t_{l}\right)}\right) \mathrm{d} t_{L-1}
$$

upon the following notations:

$$
\begin{aligned}
\operatorname{pr}(c) & =\left\{t_{L-1} \mid\left(t_{1}, \ldots, t_{L-2}, t_{L-1}\right) \in c\right\} \\
c \mid t_{L-1} & =\left\{\left(t_{1}, \ldots, t_{L-2}\right) \mid\left(t_{1}, \ldots, t_{L-2}, t_{L-1}\right) \in c\right\}
\end{aligned}
$$


Namely $f_{k}(w)(1 \leq k \leq L-1)$ is written as the Stieltjes transform

$$
f_{k}(w)=\int_{\operatorname{pr}(c)} \frac{\mathrm{d} \mu_{k}(s)}{1-w s}
$$

of a function $\mu_{k}=\mu_{k}(s)$.

In parallel, we introduce the functions

$$
h_{j}^{0}=\int_{c} \frac{t_{L-1}^{j} U(\boldsymbol{t}) \mathrm{d} t_{1} \cdots \mathrm{d} t_{L-1}}{\prod_{l=1}^{L-1}\left(t_{l-1}-t_{l}\right)}
$$

also. We thus see that

$$
h_{j}^{0}=\int_{\operatorname{pr}(c)} s^{j} \mathrm{~d} \mu_{0}(s)
$$

where

$$
\mathrm{d} \mu_{0}\left(t_{L-1}\right)=\left(\int_{c \mid t_{L-1}} \frac{U(\boldsymbol{t}) \mathrm{d} t_{1} \cdots \mathrm{d} t_{L-2}}{\prod_{l=1}^{L-1}\left(t_{l-1}-t_{l}\right)}\right) \mathrm{d} t_{L-1} .
$$

Lemma 5.1. The following linear relations (contiguity relations) hold:

$$
\begin{aligned}
h_{j}^{1}+h_{j}^{2}+\cdots+h_{j}^{L-1} & =h_{j}^{0}-h_{j+1}^{0}, \\
h_{j}^{k}-x_{i} h_{j+1}^{k} & =\ell_{i}\left(h_{j}^{k}\right),
\end{aligned}
$$

where $\ell_{i}$ denotes the down-shift operator with respect to $\beta_{i}$ defined by

$$
\ell_{i}\left(\beta_{i}\right)=\beta_{i}-1 \quad \text { and } \quad \ell_{i}\left(\beta_{j}\right)=\beta_{j} \quad(i \neq j) .
$$

Proof. By definitions (5.1) and (5.2) it is immediate to verify these formulae.

Remark 5.2. If $c$ is chosen to be the $(L-1)$-simplex (4.14):

$$
\left\{0 \leq t_{L-1} \leq \cdots \leq t_{2} \leq t_{1} \leq 1\right\} \subset \mathbb{R}^{L-1},
$$

both (5.1) and (5.2) are written by the hypergeometric function $F_{L, N}$. Let us introduce the function

$$
h=h\left[\begin{array}{c}
\boldsymbol{\alpha}, \boldsymbol{\beta} \\
\boldsymbol{\gamma}
\end{array} ; \boldsymbol{x}\right]=\prod_{l=1}^{L-1} \frac{\Gamma\left(\alpha_{l}\right) \Gamma\left(\gamma_{l}-\alpha_{l}\right)}{\Gamma\left(\gamma_{l}\right)} \times F_{L, N}\left[\begin{array}{c}
\boldsymbol{\alpha}, \boldsymbol{\beta} \\
\boldsymbol{\gamma}
\end{array} ; \boldsymbol{x}\right] .
$$

Then it holds by definition that

$$
h_{j}^{k}=h\left[\begin{array}{c}
\alpha_{1}+j+1, \ldots, \alpha_{k-1}+j+1, \alpha_{k}+j, \ldots, \alpha_{L-1}+j, \boldsymbol{\beta} \\
\gamma_{1}+j+1, \ldots, \gamma_{k}+j+1, \gamma_{k+1}+j, \ldots, \gamma_{L-1}+j
\end{array} ; \boldsymbol{x}\right] \quad(0 \leq k \leq L-1) .
$$

For example we have

$$
\begin{aligned}
& h_{j}^{0}=h\left[\begin{array}{c}
\alpha_{1}+j, \ldots, \alpha_{L-1}+j, \boldsymbol{\beta} \\
\gamma_{1}+j, \ldots, \gamma_{L-1}+j
\end{array} ; \boldsymbol{x}\right], \\
& h_{j}^{1}=h\left[\begin{array}{c}
\alpha_{1}+j, \ldots, \alpha_{L-1}+j, \boldsymbol{\beta} \\
\gamma_{1}+j+1, \gamma_{2}+j, \ldots, \gamma_{L-1}+j
\end{array} ; \boldsymbol{x}\right], \\
& h_{j}^{2}=h\left[\begin{array}{c}
\alpha_{1}+j+1, \alpha_{2}+j, \ldots, \alpha_{L-1}+j, \boldsymbol{\beta} \\
\gamma_{1}+j+1, \gamma_{2}+j+1, \gamma_{3}+j, \ldots, \gamma_{L-1}+j
\end{array} ; \boldsymbol{x}\right]
\end{aligned}
$$

and so forth. 
It is convenient to prepare the notation of the block-Toeplitz determinant (cf. Remark 2.2) for any $L$-tuple of nonnegative integers:

$$
\boldsymbol{n}=\left(n_{0}, n_{1}, \ldots, n_{L-1}\right) \in\left(\mathbb{Z}_{\geq 0}\right)^{L} .
$$

Let $|\boldsymbol{n}|=\sum_{i=0}^{L-1} n_{i}$. We set

$$
\begin{aligned}
\Delta^{(k)}(\boldsymbol{n}) & :=\operatorname{det}[\underbrace{A_{n_{0}}^{0}\left(|\boldsymbol{n}|, n_{0}\right) \cdots A_{n_{k-1}}^{k-1}\left(|\boldsymbol{n}|, n_{k-1}\right)}_{k \text { blocks }} \underbrace{A_{n_{k}-1}^{k}\left(|\boldsymbol{n}|, n_{k}\right) \cdots A_{n_{L-1}-1}^{L-1}\left(|\boldsymbol{n}|, n_{L-1}\right)}_{L-k \text { blocks }}] \\
& {\left[\begin{array}{c}
A_{|\boldsymbol{n}|}^{0}\left(n_{0},|\boldsymbol{n}|\right) \\
\vdots \\
A_{|\boldsymbol{n}|}^{k-1}\left(n_{k-1},|\boldsymbol{n}|\right) \\
A_{|\boldsymbol{n}|-1}^{k}\left(n_{k},|\boldsymbol{n}|\right) \\
\vdots \\
A_{|\boldsymbol{n}|-1}^{L-1}\left(n_{L-1},|\boldsymbol{n}|\right)
\end{array}\right] }
\end{aligned}
$$

for each $k(0 \leq k \leq L)$. If $\boldsymbol{n}=\mathbf{0}=(0, \ldots, 0)$, we fix $\Delta^{(k)}(\mathbf{0})=1$. As well as (2.2), the symbol $A_{j}^{i}(k, l)$ denotes the $k \times l$ rectangular Toeplitz matrix for the sequence $\left\{h_{j}^{i}\right\}_{j=0}^{\infty}$ whose top left corner is $h_{j}^{i}$, and $h_{j}^{i}=0$ if $j<0$. The second equality in (5.5) can be verified easily from (2.3). Henceforth we suppose

$$
\boldsymbol{n}=(0, n, \ldots, n)
$$

unless expressly stated otherwise. We will often abbreviate $\Delta^{(k)}(\boldsymbol{n})$ for $\boldsymbol{n}=(0, n, \ldots, n)$ as $\Delta^{(k)}$. Note that this convention is consistent with the description in Remark 2.2. We also prepare the canonical basis $\left\{\boldsymbol{e}_{0}, \boldsymbol{e}_{1}, \ldots, \boldsymbol{e}_{L-1}\right\}$ of $\mathbb{Z}^{L}$, i.e.

$$
\boldsymbol{e}_{k}=(0, \ldots, 0, \stackrel{k}{1}, 0, \ldots, 0)^{k}
$$

As seen in (4.15), the coefficient

$$
A(z)=\sum_{i=0}^{N+1} \frac{A_{i}}{z-u_{i}} \quad\left(u_{0}=1, u_{N+1}=0\right)
$$

of the Fuchsian system $\mathcal{L}_{L, N}$ attached to the hypergeometric solution of $\mathcal{H}_{L, N}$ with $\kappa_{0}-\sum_{i=1}^{N} \theta_{i}=0$ (see Theorem 4.3) is expressed as $A_{i}=\boldsymbol{b}^{(i)} \boldsymbol{c}^{(i)}(0 \leq i \leq N)$, where

$$
\begin{aligned}
& \boldsymbol{b}^{(0)}=h_{0}^{0} \cdot{ }^{\mathrm{T}}\left(0, \kappa_{1}, \kappa_{2}, \ldots, \kappa_{L-1}\right), \quad \boldsymbol{c}^{(0)}=\left(1, \frac{-1}{h_{0}^{0}}, \ldots, \frac{-1}{h_{0}^{0}}\right), \quad \text { and } \\
& \boldsymbol{b}^{(i)}=-\theta_{i} \cdot{ }^{\mathrm{T}}\left(1, \ell_{i}^{-1}\left(h_{0}^{1}\right), \ell_{i}^{-1}\left(h_{0}^{2}\right), \ldots, \ell_{i}^{-1}\left(h_{0}^{L-1}\right)\right), \quad \boldsymbol{c}^{(i)}=(1,0, \ldots, 0) \quad \text { for } 1 \leq i \leq N .
\end{aligned}
$$

Cf. (4.8). Our next task is applying the Schlesinger transformation to this Fuchsian system. 


\subsection{Calculation of the Schlesinger transform (I)}

To derive the action of the Schlesinger transformation, we need basically to deal with (1.10):

$$
A \mapsto \hat{A}=\sum_{i=0}^{N+1} \frac{\hat{A_{i}}}{z-u_{i}}=R A R^{-1}+\frac{\mathrm{d} R}{\mathrm{~d} z} R^{-1} .
$$

Namely, since both $R=R(z)$ and $R^{-1}$ are polynomials in $z$, each residue matrix $\hat{A}_{i}$ can be calculated by

$$
\hat{A_{i}}=R\left(u_{i}\right) A_{i} R^{-1}\left(u_{i}\right) \text { for } 0 \leq i \leq N+1 .
$$

However, thanks to (4.5), it is rather easy to calculate the diagonal parts even in the general case. First we will demonstrate it.

The multiplier $R=R(z)$ of the Schlesinger transformation (see Theorem 1.6) can be written, a little more specifically than (1.9), as

$$
R=w^{-n}\left(\left[\begin{array}{ccccc}
0 & & & & \\
* & Q_{1}^{(1)}(0) & & & \\
* & * & Q_{2}^{(2)}(0) & & \\
\vdots & \vdots & \ddots & \ddots & \\
* & * & \ldots & * & Q_{L-1}^{(L-1)}(0)
\end{array}\right]+O(w)\right)
$$

recall (1.5). Multiplying

$$
\Phi=\left(\left[\begin{array}{ccc}
1 & & \\
\vdots & \ddots & \\
* & \cdots & 1
\end{array}\right]+O(w)\right) \Xi
$$

by $R$ from the left yields $R \Phi=\hat{\Phi} \cdot \operatorname{diag}\left(w^{n(L-1)}, w^{-n}, \ldots, w^{-n}\right)$ with

$$
\hat{\Phi}=\left(\left[\begin{array}{ccc}
1 & & \\
\vdots & \ddots & \\
* & \cdots & 1
\end{array}\right]+O(w)\right) \hat{\Xi}
$$

as shown in Theorem 1.6 , where both $\Xi$ and $\hat{\Xi}$ are diagonal matrices independent of $w=1 / z$. We mention, without fear of repetition, that the Hermite-Padé approximation condition (1.1) assures

$$
R \boldsymbol{f}=O\left(w^{n(L-1)}\right)=\left[\begin{array}{c}
\rho_{n L}^{0} \\
\rho_{n L}^{1} \\
\vdots \\
\rho_{n L}^{L-1}
\end{array}\right] w^{n(L-1)}+(\text { terms of higher order })
$$

with $\boldsymbol{f}={ }^{\mathrm{T}}\left(f_{0}, \ldots, f_{L-1}\right)$ denoting the first column of $\Phi$. We thus find the formula

$$
\begin{aligned}
\hat{\Xi} \Xi^{-1} & =\operatorname{diag}\left(\rho_{n L}^{0}, Q_{1}^{(1)}(0), Q_{2}^{(2)}(0), \ldots, Q_{L-1}^{(L-1)}(0)\right) \\
& =(-1)^{n} \cdot \operatorname{diag}\left((-1)^{n L} \frac{\Delta^{(L)}}{\Delta^{(1)}}, \frac{\Delta^{(1)}}{\Delta^{(2)}}, \frac{\Delta^{(2)}}{\Delta^{(3)}}, \ldots, \frac{\Delta^{(L-1)}}{\Delta^{(L)}}\right)
\end{aligned}
$$


by virtue of Remark 2.2. Combining this with (4.5) under $x_{i}=1 / u_{i}$ :

$$
\left(A_{i}\right)_{\mathrm{D}}=x_{i} \frac{\partial}{\partial x_{i}} \log \Xi
$$

we arrive at the formulae

$$
\begin{aligned}
\left(\hat{A}_{i}\right)_{\mathrm{D}}-\left(A_{i}\right)_{\mathrm{D}} & =x_{i} \frac{\partial}{\partial x_{i}} \log \operatorname{diag}\left(\frac{\Delta^{(L)}}{\Delta^{(1)}}, \frac{\Delta^{(1)}}{\Delta^{(2)}}, \ldots, \frac{\Delta^{(L-1)}}{\Delta^{(L)}}\right) \\
& =x_{i} \operatorname{diag}\left(\frac{\mathcal{D}_{i} \Delta^{(L)} \cdot \Delta^{(1)}}{\Delta^{(L)} \Delta^{(1)}}, \frac{\mathcal{D}_{i} \Delta^{(1)} \cdot \Delta^{(2)}}{\Delta^{(1)} \Delta^{(2)}}, \ldots, \frac{\mathcal{D}_{i} \Delta^{(L-1)} \cdot \Delta^{(L)}}{\Delta^{(L-1)} \Delta^{(L)}}\right)
\end{aligned}
$$

for $1 \leq i \leq N$, where $\mathcal{D}_{i}$ denotes the Hirota differential with respect to $\partial / \partial x_{i}$.

Next we turn to the particular case, i.e. the Fuchsian system $\mathcal{L}_{L, N}$ upon the substitution (4.15) corresponding to the hypergeometric solution (see Theorem 4.3). Write the residue matrices as $\hat{A}_{i}=\hat{\boldsymbol{b}}^{(i)} \hat{\boldsymbol{c}}^{(i)}(0 \leq i \leq N)$. In order to reconstruct the canonical variables $\left(\hat{q}_{k}^{(i)}, \hat{p}_{k}^{(i)}\right)$ of the Schlesinger transform of $\mathcal{H}_{L, N}$, it is only necessary to know the quantities

$$
\hat{\boldsymbol{c}}^{(i)}=\left(\hat{c}_{k}^{(i)}\right)_{0 \leq k \leq L-1}=\left(1, \hat{c}_{1}^{(i)}, \ldots, \hat{c}_{L-1}^{(i)}\right) \quad \text { for } \quad 0 \leq i \leq N
$$

because we have already known from (5.8) the diagonal entries $\left(\hat{A}_{i}\right)_{k, k}=\hat{b}_{k}^{(i)} \hat{c}_{k}^{(i)}=-\hat{q}_{k}^{(i)} \hat{p}_{k}^{(i)}$ for $1 \leq i \leq N$; cf. (4.10). Calculations of (5.9) are a little complicated; therefore, we will separately carry out the cases $i=0$ and $i \neq 0$ in Sects. 5.3 and 5.4, respectively. The result can be found in Sect. 5.5 .

\subsection{Calculation of the Schlesinger transform (II)}

Consider the row vector $c^{(0)} R^{-1}(1)$ in view of (5.7) and $u_{0}=1$, where $c^{(0)}=\left(1,-1 / h_{0}^{0}, \ldots,-1 / h_{0}^{0}\right)$; recall (5.6).

(i) The 0th component It follows from the determinantal representation of the polynomial $P_{0}^{(0)}(w)$ and $P_{l}^{(0)}(w)=\left[f_{l} P_{0}^{(0)}\right]_{0}^{m-1}$ for $l>0$ (see Proposition 2.3 and its sequel) that

$$
P_{l}^{(0)}(1)=\frac{1}{\mathrm{NP}^{(0)}} \operatorname{det}\left[\begin{array}{c}
h_{0}^{l}+\cdots+h_{m-1}^{l}, h_{0}^{l}+\cdots+h_{m-2}^{l}, \ldots, h_{0}^{l}+h_{1}^{l}, h_{0}^{l}, 0 \\
A_{m}^{1}(n, m+1) \\
\vdots \\
A_{m}^{L-1}(n, m+1)
\end{array}\right] .
$$

Summation over $l=1,2, \ldots, L-1$ of this formula entails

$$
\sum_{l=1}^{L-1} P_{l}^{(0)}(1)=\frac{1}{\mathrm{NP}^{(0)}} \operatorname{det}\left[\begin{array}{c}
h_{0}^{0}-h_{m}^{0}, h_{0}^{0}-h_{m-1}^{0}, \ldots, h_{0}^{0}-h_{2}^{0}, h_{0}^{0}-h_{1}^{0}, 0 \\
A_{m}^{1}(n, m+1) \\
\vdots \\
A_{m}^{L-1}(n, m+1)
\end{array}\right]
$$


via the contiguity relation (5.3). Hence the 0 th component of $c^{(0)} R^{-1}(1)$ reads

$$
P_{0}^{(0)}(1)-\frac{1}{h_{0}^{0}} \sum_{l=1}^{L-1} P_{l}^{(0)}(1)=\frac{1}{h_{0}^{0} \mathrm{NP}^{(0)}} \operatorname{det}\left[\begin{array}{c}
h_{m}^{0}, h_{m-1}^{0}, \ldots, h_{0}^{0} \\
A_{m}^{1}(n, m+1) \\
\vdots \\
A_{m}^{L-1}(n, m+1)
\end{array}\right]=\frac{\Delta^{(0)}\left(\boldsymbol{n}+\boldsymbol{e}_{0}\right)}{h_{0}^{0} \Delta^{(L)}(\boldsymbol{n})}
$$

Here we have used (2.7).

(ii) The $k(>0)$ th component Similarly it holds for $l>0$ that

$$
P_{l}^{(k)}(1)=\frac{1}{\mathrm{NP}^{(k)}} \operatorname{det}\left[\begin{array}{c}
h_{0}^{l}+\cdots+h_{m-1}^{l}, h_{0}^{l}+\cdots+h_{m-2}^{l}, \ldots, h_{0}^{l}+h_{1}^{l}, h_{0}^{l} \\
A_{m}^{1}(n, m) \\
\vdots \\
A_{m}^{k-1}(n, m) \\
A_{m}^{k}(n-1, m) \\
A_{m-1}^{k+1}(n, m) \\
\vdots \\
A_{m-1}^{L-1}(n, m)
\end{array}\right] .
$$

Taking a sum and using (5.3) thus yield

$$
\begin{aligned}
P_{0}^{(k)}(1)-\frac{1}{h_{0}^{0}} \sum_{l=1}^{L-1} P_{l}^{(k)}(1) & =\frac{1}{h_{0}^{0} \mathrm{NP}^{(k)}} \operatorname{det}\left[\begin{array}{c}
h_{m}^{0}, h_{m-1}^{0}, \ldots, h_{1}^{0} \\
A_{m}^{1}(n, m) \\
\vdots \\
A_{m}^{k-1}(n, m) \\
A_{m}^{k}(n-1, m) \\
A_{m-1}^{k+1}(n, m) \\
\vdots \\
A_{m-1}^{L-1}(n, m)
\end{array}\right] \\
& =\frac{(-1)^{n k+1} \Delta^{(k+1)}\left(\boldsymbol{n}+\boldsymbol{e}_{0}-\boldsymbol{e}_{k}\right)}{h_{0}^{0} \Delta^{(k)}(\boldsymbol{n})} .
\end{aligned}
$$

Here we have used (2.8).

The ratio of (5.10) and (5.11) leads to the formula

$$
\hat{c}_{k}^{(0)}=(-1)^{n k+1} \frac{\Delta^{(L)}(\boldsymbol{n}) \Delta^{(k+1)}\left(\boldsymbol{n}+\boldsymbol{e}_{0}-\boldsymbol{e}_{k}\right)}{\Delta^{(k)}(\boldsymbol{n}) \Delta^{(0)}\left(\boldsymbol{n}+\boldsymbol{e}_{0}\right)} .
$$

\subsection{Calculation of the Schlesinger transform (III)}

Consider for $1 \leq i \leq N$ the row vector $c^{(i)} R^{-1}\left(u_{i}\right)$ in view of (5.7) and $u_{i}=1 / x_{i}$, which is nothing but the top row of the matrix $R^{-1}\left(u_{i}\right)$ due to $c^{(i)}=(1,0, \ldots, 0)$; recall (5.6). 
(i) The 0th component We are interested in the following determinant

$$
x_{i}^{-m} P_{0}^{(0)}\left(x_{i}\right)=\frac{x_{i}^{-m}}{\mathrm{NP}^{(0)}} \operatorname{det}\left[\begin{array}{c}
1, x_{i}, \ldots, x_{i}^{m} \\
A_{m}^{1}(n, m+1) \\
\vdots \\
A_{m}^{L-1}(n, m+1)
\end{array}\right] \text {. }
$$

Subtracting the $j$ th column multiplied by $x_{i}$ from the $(j+1)$ th column for $j=m, m-1, \ldots, 1$ sequentially and using the contiguity relation (5.4), we thus obtain

$$
\begin{aligned}
x_{i}^{-m} P_{0}^{(0)}\left(x_{i}\right) & =\frac{x_{i}^{-m}}{\operatorname{NP}^{(0)}} \operatorname{det}\left[\begin{array}{cc}
1 & 0, \ldots, 0 \\
A_{m}^{1}(n, 1) & \ell_{i}\left(A_{m-1}^{1}(n, m)\right) \\
\vdots & \vdots \\
A_{m}^{L-1}(n, 1) & \ell_{i}\left(A_{m-1}^{L-1}(n, m)\right)
\end{array}\right]=\frac{x_{i}^{-m}}{\operatorname{NP}^{(0)}} \ell_{i}\left(\operatorname{det}\left[\begin{array}{c}
A_{m-1}^{1}(n, m) \\
\vdots \\
A_{m-1}^{L-1}(n, m)
\end{array}\right]\right) \\
& =\frac{(-1)^{m} x_{i}^{-m} \ell_{i}\left(\Delta^{(0)}(n)\right)}{\Delta^{(L)}(\boldsymbol{n})} .
\end{aligned}
$$

(ii) The $k(>0)$ th component In the same way as above, we find

$$
\begin{aligned}
x_{i}^{-m+1} P_{0}^{(k)}\left(x_{i}\right) & =\frac{x_{i}^{-m+1}}{\mathrm{NP}^{(k)}} \operatorname{det}\left[\begin{array}{c}
1, x_{i}, \ldots, x_{i}^{m-1} \\
A_{m}^{1}(n, m) \\
\vdots \\
A_{m}^{k-1}(n, m) \\
A_{m}^{k}(n-1, m) \\
A_{m-1}^{k+1}(n, m) \\
\vdots \\
A_{m-1}^{L-1}(n, m)
\end{array}\right]=\frac{x_{i}^{-m+1}}{\mathrm{NP}^{(k)}} \ell_{i} \\
= & \frac{(-1)^{n(L-k-1)} x_{i}^{-m+1} \ell_{i}\left(\Delta^{(k+1)}\left(\boldsymbol{n}-\boldsymbol{e}_{k}\right)\right)}{\Delta^{(k)}(\boldsymbol{n})}
\end{aligned}
$$

Hence we verify from the ratio of (5.12) and (5.13) that

$$
\hat{c}_{k}^{(i)}=(-1)^{n k} x_{i} \frac{\Delta^{(L)}(\boldsymbol{n}) \ell_{i}\left(\Delta^{(k+1)}\left(\boldsymbol{n}-\boldsymbol{e}_{k}\right)\right)}{\Delta^{(k)}(\boldsymbol{n}) \ell_{i}\left(\Delta^{(0)}(\boldsymbol{n})\right)} .
$$

\subsection{Result}

Summarizing the above we are led to the following result. 
Theorem 5.3. Let $n$ be a positive integer. If $\kappa_{0}-\sum_{i=1}^{N} \theta_{i}=n(L-1)$ then the Hamiltonian system $\mathcal{H}_{L, N}$ possesses a solution $\left(\hat{q}_{k}^{(i)}, \hat{p}_{k}^{(i)}\right)$ given by

$$
\begin{aligned}
\hat{q}_{k}^{(i)} & =-x_{i} \frac{\Delta^{(0)}\left(\boldsymbol{n}+\boldsymbol{e}_{0}\right) \ell_{i}\left(\Delta^{(k+1)}\left(\boldsymbol{n}-\boldsymbol{e}_{k}\right)\right)}{\Delta^{(k+1)}\left(\boldsymbol{n}+\boldsymbol{e}_{0}-\boldsymbol{e}_{k}\right) \ell_{i}\left(\Delta^{(0)}(\boldsymbol{n})\right)}, \\
\hat{q}_{k}^{(i)} \hat{p}_{k}^{(i)} & =-x_{i} \frac{\mathcal{D}_{i} \Delta^{(k)}(\boldsymbol{n}) \cdot \Delta^{(k+1)}(\boldsymbol{n})}{\Delta^{(k)}(\boldsymbol{n}) \Delta^{(k+1)}(\boldsymbol{n})}
\end{aligned}
$$

under the correspondence

$$
\alpha_{k}=e_{k}-e_{0}, \quad \beta_{i}=-\theta_{i}, \quad \gamma_{k}=e_{k}-e_{0}-\kappa_{k}+n
$$

of constant parameters, where $\boldsymbol{n}=(0, n, \ldots, n) \in\left(\mathbb{Z}_{\geq 0}\right)^{L}$.

Remark 5.4. We have in fact an alternative expression

$$
\hat{q}_{k}^{(i)} \hat{p}_{k}^{(i)}=\theta_{i} x_{i} \frac{\ell_{i}^{-1}\left(\Delta^{(k)}\left(\boldsymbol{n}+\boldsymbol{e}_{k}\right)\right) \ell_{i}\left(\Delta^{(k+1)}\left(\boldsymbol{n}-\boldsymbol{e}_{k}\right)\right)}{\Delta^{(k)}(\boldsymbol{n}) \Delta^{(k+1)}(\boldsymbol{n})}
$$

of the above solution with no use of the Hirota differentials. This formula can be verified by calculating $\hat{\boldsymbol{b}}^{(i)}$ in the same manner as Sects. 5.3 and 5.4; further details might be left to the reader.

As seen in Theorem 5.3, we have constructed a particular solution of $\mathcal{H}_{L, N}$ expressed in terms of the block-Toeplitz determinant $\Delta^{(k)}(\boldsymbol{n})$ whose entries are given by the hypergeometric functions. Finally we shall rewrite $\Delta^{(k)}(\boldsymbol{n})$ as an iterated hypergeometric integral: if we remember that $h_{j}^{i}$ is defined as a moment (see Sect. 5.1), then we observe that

$$
\begin{aligned}
& \Delta^{(k)}(\boldsymbol{n})=\int \operatorname{det}\left[\left[s_{0, j}{ }^{n_{0}+i-j}\right]_{\substack{1 \leq i \leq|\boldsymbol{n}| \\
1 \leq j \leq n_{0}}}, \ldots,\left[s_{k-1, j}{ }^{n_{k-1}+i-j}\right]_{\substack{1 \leq i \leq|\boldsymbol{n}| \\
1 \leq j \leq n_{k-1}}},\left[s_{k, j}{ }^{n_{k}+i-j-1}\right]_{\substack{1 \leq i \leq|\boldsymbol{n}| \\
1 \leq j \leq n_{k}}}, \ldots,\left[s_{L-1, j}{ }^{n_{L-1}+i-j-1}\right]_{\substack{1 \leq i \leq|\boldsymbol{n}| \\
1 \leq j \leq n_{L-1}}}\right] \\
& \times \prod_{a=0}^{L-1} \prod_{j=1}^{n_{a}} \mathrm{~d} \mu_{a}\left(s_{a, j}\right) \\
& =\int \operatorname{det}\left[\left[s_{0, j}{ }^{i-1}\right]_{\substack{1 \leq i \leq|\boldsymbol{n}| \\
1 \leq j \leq n_{0}}},\left[s_{1, j}{ }^{i-1}\right]_{\substack{1 \leq i \leq|\boldsymbol{n}| \\
1 \leq j \leq n_{1}}}, \ldots,\left[s_{L-1, j}{ }^{i-1}\right]_{\substack{1 \leq i \leq|\boldsymbol{n}| \\
1 \leq j \leq n_{L-1}}}\right] \prod_{a=0}^{k-1} \prod_{j=1}^{n_{a}} s_{a, j} \\
& \times \prod_{a=0}^{L-1} \prod_{j=1}^{n_{a}} s_{a, j}{ }^{n_{a}-j} \mathrm{~d} \mu_{a}\left(s_{a, j}\right)
\end{aligned}
$$

through Fubini's theorem. The Vandermonde determinant shows that

$$
\operatorname{det}\left[\left[s_{0, j}^{i-1}\right]_{\substack{1 \leq i \leq|\boldsymbol{n}| \\ 1 \leq j \leq n_{0}}},\left[s_{1, j}^{i-1}\right]_{\substack{1 \leq i \leq \boldsymbol{n} \mid \\ 1 \leq j \leq n_{1}}}, \ldots,\left[s_{L-1, j}{ }^{i-1}\right]_{\substack{1 \leq i \leq|\boldsymbol{n}| \\ 1 \leq j \leq n_{L-1}}}\right]=\prod_{(a, b)>(c, d)}\left(s_{a, b}-s_{c, d}\right),
$$

where $(a, b)>(c, d)$ is defined to mean

$$
" a>c " \text { or " } a=c \text { and } b>d " .
$$


Also it holds that

$$
\sum_{\sigma \in \operatorname{Sym}\left(n_{a}\right)} \operatorname{sgn} \sigma \prod_{j=1}^{n_{a}} s_{a, \sigma(j)}{ }^{n_{a}-j}=\prod_{b<c}\left(s_{a, b}-s_{a, c}\right),
$$

where $\operatorname{Sym}\left(n_{a}\right)$ denotes the symmetric group on $\left\{1,2, \ldots, n_{a}\right\}$. Therefore, because the value of integral (5.14) is invariant under a permutation of the variables $\left\{s_{a, b}\right\}_{1 \leq b \leq n_{a}}$ for each $0 \leq a \leq L-1$, we conclude that

$$
\Delta^{(k)}(\boldsymbol{n})=\prod_{a=0}^{L-1} \frac{1}{n_{a} !} \int\left(\prod_{a=0}^{k-1} \prod_{j=1}^{n_{a}} s_{a, j}\right)\left(\prod_{(a, b)>(c, d)}\left(s_{a, b}-s_{c, d}\right)\right) \prod_{a=0}^{L-1}\left(\prod_{b<c}\left(s_{a, b}-s_{a, c}\right)\right) \prod_{j=1}^{n_{a}} \mathrm{~d} \mu_{a}\left(s_{a, j}\right) .
$$

\section{A Verification of (4.4)}

Usually, we often normalize the Fuchsian system (4.1) so that $A_{N+2}=-\sum_{i=0}^{N+1} A_{i}$ (the residue matrix at $z=\infty$ ) is diagonal when considering its isomonodromic deformation; see e.g. [10]. But, in this paper, we adopt a different normalization treating the two points $z=0$ and $z=\infty$ equally; i.e. we choose $A_{N+1}$ (the residue matrix at $z=0$ ) and $A_{N+2}$ to be upper and lower triangular, respectively. Note that the latter normalization is more versatile in a general setting than the former. In this appendix, for a supplement to Sect.4.1, we demonstrate how to determine the coefficient $B_{i}=B_{i}(z)$ of the deformation equation (4.2):

$$
\frac{\partial Y}{\partial u_{i}}=B_{i} Y
$$

of (4.1).

Differentiating (4.3) with respect to $u_{j}$ tells us that

- if $i \neq j$ then $\frac{\partial Y}{\partial u_{j}} Y^{-1}$ is holomorphic at $z=u_{i}$;

- if $i=j$ then $\frac{\partial Y}{\partial u_{i}} Y^{-1}=-\frac{A_{i}}{z-u_{i}}+\left(\right.$ holomorphic at $\left.z=u_{i}\right)$.

Similarly, we observe that

$$
\frac{\partial Y}{\partial u_{i}} Y^{-1}=\frac{\partial \Psi}{\partial u_{i}} \Psi^{-1}=\left[\begin{array}{cccc}
0 & * & \cdots & * \\
& 0 & \ddots & \vdots \\
& & \ddots & * \\
& & & 0
\end{array}\right]+O(z) \quad \text { near } z=0
$$

and

$$
\frac{\partial Y}{\partial u_{i}} Y^{-1}=\frac{\partial \Phi}{\partial u_{i}} \Phi^{-1}=\left[\begin{array}{ccc}
* & & \\
\vdots & \ddots & \\
* & \cdots & *
\end{array}\right]+O(w) \quad \text { near } z=1 / w=\infty
$$

Here we have used the assumption that the connection matrix $C$ does not depend on $u_{i}$. Consequently, $B_{i}=\frac{\partial Y}{\partial u_{i}} Y^{-1}$ is a rational function matrix in $z$ that has only a simple pole at $z=u_{i}$ with residue $-A_{i}$ and thus

$$
B_{i}=\frac{A_{i}}{u_{i}-z}+K_{i},
$$


where $K_{i}$ is a constant matrix. Substituting $z=\infty(w=0)$ in (A.2) shows that $K_{i}$ is a lower triangular matrix. Therefore, substituting $z=0$ in (A.1), we conclude that $K_{i}=-\left(A_{i}\right)_{\mathrm{LT}} / u_{i}$.

Acknowledgement. The authors are deeply grateful to Shuhei Kamioka for giving them an exposition on various multi-dimensional continued fractions. They appreciate Satoshi Tsujimoto his kind information about literature on rational approximations. Also, they have benefited from invaluable discussions with Yasuhiko Yamada. This work was supported in part by a grant-in-aid from the Japan Society for the Promotion of Science (Grant Number: 25800082 and 25870234).

\section{References}

[1] Aomoto, K., Kita, M.: Theory of Hypergeometric Functions. Springer, Tokyo (2011)

[2] Baker, G., Graves-Morris, P.: Padé approximants, 2nd edn. Cambridge University Press, Cambridge (1996)

[3] Chudnovsky, D.V., Chudnovsky, G.V.: Bäcklund transformations for linear differential equations and Padé approximations. I. J. Math. Pures Appl. 61, 1-16 (1982)

[4] Chudnovsky, D.V., Chudnovsky, G.V.: Explicit continued fractions and quantum gravity. Acta Appl. Math. 36, 167-185 (1994)

[5] Coates, J.: On the algebraic approximation of functions. I. Indag. Math. 28, 421-434 (1966)

[6] Haraoka, Y.: Regular coordinates and reduction of deformation equations for Fuchsian systems. In: Balser, W., Filipuk, G., Łysik, G., Michalik, S. (Eds.), Formal and Analytic Solutions of Differential and Difference Equations, pp. 39-58. Polish Acad. Sci. Inst. Math., Warsaw (2012)

[7] Ikawa, Y.: Hypergeometric solutions for the $q$-Painlevé equation of type $E_{6}^{(1)}$ by the Padé method. Lett. Math. Phys. 103, 743-763 (2013)

[8] Ishikawa, M., Mano, T., Tsuda, T.: Determinant structure for $\tau$-function of holonomic deformation of linear differential equations. (in preparation)

[9] Jimbo, M., Miwa, T.: Monodromy perserving deformation of linear ordinary differential equations with rational coefficients. II. Physica D 2, 407-448 (1981)

[10] Jimbo, M., Miwa, T., Ueno, K.: Monodromy perserving deformation of linear ordinary differential equations with rational coefficients. I. Physica D 2, 306-352 (1981)

[11] Jones, W.B., Thron, W.J.: Continued Fractions: Analytic Theory and Applications, reissue edn. Cambridge University Press, Cambridge (2009)

[12] Kato, Y., Aomoto, K.: Jacobi-Perron algorithms, bi-orthogonal polynomials and inverse scattering problems. Publ. Res. Inst. Math. Sci. 20, 635-658 (1984)

[13] Laguerre, E.: Sur la réduction en fractions continues d'une fonction qui satisfait à une équation linéaire du premier ordre à coefficients rationnels. Bull. Soc. Math. France 8, 21-27 (1880)

[14] Magnus, A.P.: Painlevé-type differential equations for the recurrence coefficients of semi-classical orthogonal polynomials. J. Comput. Appl. Math. 57, 215-237 (1995) 
[15] Mahler, K.: Perfect systems. Compos. Math. 19, 95-166 (1968)

[16] Mano, T.: Determinant formula for solutions of the Garnier system and Padé approximation. J. Phys. A: Math. Theor. 45, 135206 (14pp) (2012)

[17] Nagao, H.: The Padé interpolation method applied to $q$-Painlevé equations. Lett. Math. Phys. 105, 503-521 (2015)

[18] Nikishin, E.M., Sorokin, V.N.: Rational approximations and orthogonality. Amer. Math. Soc., Providence, RI (1991)

[19] Noumi, M., Tsujimoto, S., Yamada, Y.: Padé interpolation for elliptic Painlevé equation. In: Iohara, K., Morier-Genoud, S., Rémy, B. (Eds.), Symmetries, Integrable Systems and Representations, pp. 463-482. Springer, London (2013)

[20] Parusnikov, V.I.: The Jacobi-Perron algorithm and simultaneous approximation of functions. Math. USSR Sb. 42, 287-296 (1982)

[21] Perron, O.: Grundlagen für eine Theorie des Jacobischen Kettenbruchalgorithmus. Math. Ann. 64, $1-76(1907)$

[22] Schlesinger, L.: Über eine klasse von differentialsystemen beliebiger ordnung mit festen kritischen punkten. J. reine angew. Math. 141, 96-145 (1912)

[23] Tsuda, T.: Universal characters and an extension of the KP hierarchy. Comm. Math. Phys. 248, 501$526(2004)$

[24] Tsuda, T.: From KP/UC hierarchies to Painlevé equations. Int. J. Math. 23, 1250010 (59pp) (2012)

[25] Tsuda, T.: Hypergeometric solution of a certain polynomial Hamiltonian system of isomonodromy type. Q. J. Math. 63, 489-505 (2012)

[26] Tsuda, T.: UC hierarchy and monodromy preserving deformation. J. reine angew. Math. 690, 1-34 (2014)

[27] Tsuda, T.: On a fundamental system of solutions of a certain hypergeometric equation. Ramanujan J. 38, 597-618 (2015)

[28] Yamada, Y.: Padé method to Painlevé equations. Funkcial. Ekvac. 52, 83-92 (2009) 